Aus Prof. L. Landau's Frauenklinik in Berlin.

\title{
Die Adenomyome der Leistengegend und des hinteren Scheidengewölbes, ihre Stellung zu den paroophoralen Adenomyomen der Uterus- und Tubenwandung v. Recklinghausen's.
}

Von

\author{
Dr. Ludwig Piek, \\ Assistenzarzt fiir pathologische Anatomie.
}

(Mit 4 Abbildungen anf Tafel XXIL 1 . XXIII nd 1 Abhildung im Text.)

Die Adenomyome und Cystadenome der Uterus- und Tubenwandung sind nach v. Recklinghausen zweifachen Ursprungs. Für die opithelialen Bestandthejle der Adenomyome und Cystadenome der Tuben, der Tubenwinkel und der peripherischen Gebärmutterschichten bilden Urnierencanälchen, wahrscheinlich paroophoraler Abkunft, die Matrix. Dagegen stammen die centralen Uterusadenomyome von der Gebärmutterschleimhaut, also vom Epithel der Müller'schen Gänge. Der Beğründung dieser Theorie hat v. Recklinghausen ein ausführliches Werk "Die Adenomyome und Cystadenome der Uterus- und Tubenwandung, ihre Abkunft von Resten des Wolff'schen Körpers" (1) ${ }^{1}$ ) gewidmet.

Die darin niedergelegten Anschauungen haben vielseitige $\mathrm{Zu-}$ stimmung, von zwei Seiten dagegen - von Kossmann (3) und von v. Lockstädt (4) — lebhaften Widerspruch gefunden. Beide Autoren erklären unter sehr eingehender und sorgfältiger Begründung die Abkunft von Epitheleinschlüssen der Adenomyome und Cystadenome der Uterus- und Tubenwand vom Wolff'schen Körper

1) Die in Paranthese geschlossenen Zahlen beziehen sich auf die Jiteraturübersicht am Ende des Aufsatzes. 
für unwahrscheinlich. Die Epithelelemente nicht nur eines Theiles, sondern aller Gesehwülste dieser Categorie seien "mit viel grösserer" (K.) oder nmit höchster Wahrscheinlichkeit" (v. L.) auf das Epithel dor Müller'schen Fäden, und zwar "auf normale oder accessorische Müller'sche Gänge" (K.) oder. „auf embryonal versprengte Epithelien". derselben (v. L.) - zurückzuführen.

Immerhin steht $v$. Lockstädt der $v$. Recklinghausen'schen Annahme nicht ganz so schroff wie Kossmann gegenüber.

Die „Schleimhauttheorie" besitzt nach v. L. zwei ganz besondere Vorzüge. Erstens ist sie für gewisse Fälle, von denen die übrigen grundsätzlich nicht zu trennen seien, direct erwiesen, und zweitens erklärt sie zwanglos die Bildung cytogenen Bindegewebes, das in vielen Adenomyomen, und zwar nur in solchen des Uterus, gefunden wird. Denn cytogenes Bindegewebe ist als regelmässiger physiologischer Bestandtheil in der Uteruskörperschleimhaut, nicht aber in den Ueberbleibseln des Wolff'schen Körpers nachgewiesen. Andererseits aber räumt v. Lockstädt ausdrücklich ein, dass die Schleimhautheorie „nicht für alle Fälle ausreicht". Die Prädilectionsstellen der Adenomyome, insbesondere aber die morphologisch eigenartigen Drüsensysteme im ersten Falle v. Recklinghausen's (1, S. 3, 96, 104, vergl. auch die Abbildungen Taf. V-IX) fänden durch v. R.'s Ableitung eine bessere Erklärung.

Dass ich mich selbst zu den Anschauungen v. Recklinghausen's von der zwiefältigen Herkunft der Adenomyome der Gebärmutter und Tube bekennen muss, habe ich auf der anatomischen Grundlage des von meinem hochverehrten Chef, Prof. L. Landau, operativ gewonnenen Materials an anderer Stelle ausfübrlich dargelegt (2). Unsere dort beschriebene Beobachtung ist betreffs ihrer Schlusskraft dem „massgebenden Fall I"v. Recklingshaus en's ebenbürtig, ja, diese beiden Fälle von Gebärmutteradenomyomen sind bisher die einzigen mit einer für die "paroophorale" Theorie v. R.'s in mikroskopischer Beziehung beweisenden Ausbeute. Die frappante Uebereinstimmung der drüsigen Bildungen in v. R.'s Beobachtung „mit dem Bauverhältniss der drüsigen Gestalten des Wolff'sehen Organs" konnte ich durch unseren Fall sogar noch in einigen Punkten vervollständigen (Nachweis von Pseudoglomeruli in Endkolbeneysten und von Flimmerepithel in peripherischen Cystchen der Geschwulst: Residuen der embryonalen Peritonealtrichter).

Durch diese von v. Recklinghausen und ron uns erhobenen Befunde in Verbindung mit meinen folgenden Ausführungen sind 
nun zwar, wie ich glaube, die Angriffe Kossmann's und v. Lockstädt's gegen die paroophorale Adenomyomtheorie in ihrer Allgemeinheit ein für alle Mal widerlegt. Ich will indessen hier im Besonderen von vornherein kurz auf einige Punkte der Ausführungen dieser Autoren eingehen, die mir auf einer missverständlichen Auffassung zu beruhen scheinen.

So handelt es sich keineswegs darum, zu Gunsten der Urnierentheorie für die Genese aller übrigen Adenomyome an Uterus und Tube die Schleimhauttheorie zu verwerfen (4, S. 38). Beide Anschauungen bestehen nicht exclusiv $(4$, S. 24, 32, 37), sondern pach v. Recklinghausen nebeneinander (z. B. 1, S. 233 u. 234). Auch ich habe in meinem früheren Aufsatz (S. 118) diese Coordination ausdrücklich betont.

Wenn Kossmann sagt, dass seine Behauptung, die Wand der Parovarialschläuche bestehe lediglich aus Bindegewebe, von v. Recklinghausen (1, S. 38) anerkannt sei (3, S. 369), so citire ich dagegen v. R.'s Ausführungen auf S. 181, nach denen „die Tunica propria der Parovarialcanälchen nicht selten glatte Muskelfasern besitzt".

Auch wird keineswegs (3, S. 365) ndie Verzweigung der Schläuche im Parovarium vermisst". Ich verweise z. B. auf die Angaben v. Recklinghausen's (1, S. 115, 117, 149) über reichliche Verästelung an den Blinddärmehen des Parovariums und des Giraldès'schen Organs.

Die von v. Recklinghausen in seinem Fall I - wie auch von uns - beobachtete höchst eigenartige Morphologie der Epithelschläuche, die zum Vergleich mit den "drüsigen Gestalten des Wolff'schen Körpers" geradezu herausfordert, thut Kossmann kurzer Hand mit der Erklärung ab (3, S. 364), "dass jede dem Müller'schen Gange angehörige Bildung, sei es ein accessorisches Gebilde, sei es eine abgesprengte Gruppe von Uterindrüsen, in denselben Gestalten auftreten kann und muss, sobald eine Entzündung oder Neubildung sie beeinflusst". Man darf für diese Behauptung wohl erst den thatsächlichen Beweis abwarten. Ich selbsthabe in sicheren schleimhäutigen Adenomyomen des Uterus mit mächtiger Drüsenwucherung und schönsten "Inseln" bisher vergeblich danach gesucht.

Und wenn endlich Kossmann hervorhebt, dass nicht entartete "Glomeruli der Urniere" - man darf sogar getrost sagen "Urnierenreste" - im nicht myomatösen Myometrium noch nie gefunden worden sind, so gilt das, wie K. übrigens selbst zugesteht 
464 Pick, Die Adenomyome der Leistengegend und des hinteren

(3, S. 378), doch genau so für den von ihm supponirten accessorischen Müller'schen Gang am Uterus. Auch einen solehen hat dort bisher noch Niemand gesehen.

Ganz besonders bemerkenswerth ist es, dass Kossmann als Hauptargument gegen $\nabla$. Recklinghausen's Theorie gerade diejenige Thatsache anführt, die für $\nabla$. R. ein wesentliches positives Beweismittel seiner Anschauung bildet (1, S. 157), nämlich die Localisation der Adenomyome. Während v. R. das Fehlen im cervicalen Myometrium, die unbedingte Bevorzugung der Dorsalwand des Uterus und der Tubenwinkel seitens der Adenomyome als eine fundamentale Stütze seiner Theorie betont, weil gerade an diesen Stellen eine embryonale Incorporation von Urnierencanälchen in den Müller'schen Gang stattfinden kann, ist nach Koss$\operatorname{mann}$ (3, S. 376; auch S. 377 u. 383) die viel grössere Häufigkeit dieser Tumoren am Uterusscheitel und in den Tubenecken in Wirklichkeit ein gewichtiger Grund gegen solche Ableitung. "Man kann selbst so weit gohen", fährt K. fort, „die Anwesenheit versprengter Urnierenreste im Myometrium des Corpus uteri und Isthmus tubae für ganz unbegreiflich zu erklären, falls nicht überhaupt eine ganz beträchtliche Missbildung der inneren Genitalien vorhanden ist." Diese Behauptung begründet Kossmann unter Anderem durch die Abbildung von 4 Querschnitten durch ein Embryon von $24 \mathrm{~mm}$ Scheitelsteisslänge (Fig. 6-10).

Ich bin eigentlich der Meinung, dass v. Recklinghausen auf S. 159 seines Werkes (vergl. auch S. 209 u.) eine genügend eindeutige Schilderung des genannten embryonalen Incorporationsvorganges gegeben hat, aus der das Irrige der Kossmann'schen Kritik in diesem Punkte ohne Weiteres hervorgeht. Da indessen auch bei v. Lockstädt (4, S. 37) augenscheinlich eine missverständliche Auffassung v. Recklinghausen's in dieser wesentlichen Frage besteht - v. L. glaubt, dass man nach v. R. die Adenomyome „am häufigsten in der abdominalen Hälfte der Tube, jedenfalls viel häufiger an der Tube selbst, als am Uterus" finden müsse - , so möchte ich hier mit einigen Worten auf den in Redo stehenden embryonalen Einschluss von Urnierencanälchen in die Substanz der Müller'schen Gänge eingehen.

Bekanntlich entwickelt sich bei allen Wirbelthieren sehr frühzeitig der Wolff'sche Körper oder die Urniere beiderseits vom Gekröse des Darmes in Gesta]t einer rom Coelomepithel überkleideten Längsfalte. Die ausgesprochen kammförmige Drüse - 
mit lateralem Hauptgang (Wolff'schem oder Urnierengang) und von medial her sich in diesen einsenkenden Quer- (Urnieren) canäIchen - springt bandartig (Umierenfalte) namentlich in ihrem hinteren voluminöseren Absehnitt gegen die Leibeshöhle vor und erstreckt sich auf dem Gipfel ihrer Ausbildung von der Lebergegend bis zum hinteren Ende der Leibeshöhle (Hertwig, 15, S. 268 und Fig. 179, S. 269). Während durch Differenzirungs- und Wucherungsprocesse des Coelomepithels auf der medialen Urnierenseite der Eierstock entsteht, bildet sich an ihrer lateralen Fläche der MüIler'sche Gang. Fine Einstülpung des verdickten Coelomepithels wächst vom proximalen Ende des Wolff'schen Körpers aus in die Tiefe, der ventralen Wand des Wolff'schen Ganges innig angeschlossen, ihm als seinem "Teitgebilde" folgend, bis zum Canalis urogenitalis hinab (Nagel, 20, S. 22 und S. 70) ${ }^{1}$ ).

So liegt der proximale Abschnitt des Müller'schen Ganges in der Urnierenfalte etwas ventral und lateral zum WoIff'schen Gang; dieser schiebt sich hier zwischen ersteren und die queren Urnierencanälchen.

Dies Lageverhältniss aber erfährt distalwärts "von Anfang $a^{*}$ (Nagel, 20, S. 71) eine Aenderung. "Die ventrale Kante der Urniere biegt durch eine halbspiralige Drehung des unteren Abschnitts des Organs nach innen, das heisst nach der Längsachse des embryonalen Körpers zu um." Damit gelangt der Müller'sche Gang, der im oberen Theil der Urnierenfalte lateral vom Wolff'schen Gang gelegen ist, „in dem unteren Theil des Organs, in der Plica urogenitalis (Waldeyer)" unter Kreuzung der Ventralwand des Wolff'schen Ganges an die mediale Seite desselben. D. h. der Müller'sche Gang rückt hier genau auf dịeselbe Seite des Wolff'sehen Ganges, von der her die Urnierencanälshen in diesen eintreten. Sind also überhaupt zu der Zeit, wo diese halbspiralige Umschlingung des W. G. durch den M. G. stattfindet, die Quercanälchen des distalen Urnierenabschnitts noch nicht völlig atrophirt, so sind an der Stelle der Kreuzung sowohl wie

1) So beschreibt Nagel (21) den Vorgang beim Menschen. Nach anderen Autoren entsteht bekanntlich der Müller'sche Gang bei Amnioten in seinem unteren Abschnitt durch Abspaltung oder Mithetheiligung aus dem Wolff'schen Gang oder sogar überhaupt -- wie bei Anamniern - durch totale Abspaltung aus diesem (vergl. Nagel, 20, S. 71, Hertwig, 15, S. 274 bis 276, Klein, 10, S. 168 u. 169). Diese Differenz der Anschaungen ist indessen für die folgenden Auseinandersetzungen ohne Belang. 
distalwärts von dieser die Bedingungen für eine Incorporation von paroophoralen oder diesen äquivalenten Urnierencanälchen in die unmittelbar benachbarte Dorsalwand des M. G. direct gegeben.

Nach der eben citirten Darstellung Nagel's erfolgt die modialwärts gerichtete Umbiegung des Müller'schen Ganges zugleich mit dem unteren Abschnitt der Urniere. Der Müller'sche Gang kreuzt also ventralwärts nicht bloss den Wolff'schen Gang, sondern den Wolff'schen Körper. Er tritt nicht bloss an die Innenseite des bereits zweiglosen Wolff'schen Ganges, sondern trifft dort auf noch ausgebildete distale Urnierencanälchen. Dies Verhalten stimmt auch sonst gut zu den entwicklungsgeschichtlichen Thatsachen. Man erwäge: Entwicklungshöhepunkt der bis zum hinteren Körperende reichenden Urniere "nach den übereinstimmenden Untersuchungen aller Forscher" bei menschlichen Embryonen von $22 \mathrm{~mm}$ Länge (6.--7. Woche) (vgl. v. Mihálcovicz, 17, Weber, 22, S. 8 und S. 9) und andererseits Ankunft des Müller'schen Ganges am Sinus urogenitalis bei Embryonen von 25-30 mm Länge, also etwa in der 7. Woche. Danach streicht der Müller'sehe Gang auf seinem halbspiraligen Weg nach abwärts anscheinend in allen Fällen an noch erhaltenen distalen Canälchen des Wolff'schen Körpers mit seinem Dorsum vorbei, und die Incorporation derselben ist danach ein vielleicht sehr häufiger, jedenfalls aber ein durchaus natürlich erscheinender Vorgang. Die Versuche, in der Tuben- oder Uteruswand von Neugeborenen und Kindern diese Einschlüsse nachzuweisen, sind zwar vor der Hand noch negativ ausgefallen ( $v$. Recklinghausen, 1, S. 151 und S. 207). Sie dürften indess wesentlich an der gewöhnlichen Neigung der distalen Urnierencanälchen zur frühzeitigen totalen Rückbildung, die auch hier an der Transpositionsstelle erfolgt, gescheitert sein und für Föten, zumal an einem grösseren Material systematisch durchgeführt, mehr Aussicht bieten.

Die andere Möglichkeit ist die, dass zu der Zeit, wo der Müllersche den Wolff'schen Gang auf seiner Wanderung nach dessen Innenseite hin kreuzt, der Canälchenschwund am distalen Ende der Urniere bereits vollendet ist, also Wolff'scher Körper einerseits und Wolff'scher Gang andererseits so differenzirt sind, wie es das beistehende bekannte Schema zeigt. Aber auch dann liegt, wie leicht ersichtlich, das Dorsum des Müller'schen Ganges genau ebenso in "unmittelbarer Nachbarschaft" von Urnierencanäl- 
Scheidengewölbes, thre Stcllung zu den paroophor. Adenomyomen ete. 467 chen ${ }^{1}$. Eine nur um ein Weniges verzögerte Involution derselben; etwa in Folge individuell kräftigerer Entwicklung des Wolff'schen Körpers oder längeren Appositionswachsthums (Peters, 23, S. 9) am distalen Urnierenende ${ }^{2}$ ) führt wiederum auch caudalwärts von der Kreuzung auf das Leichteste zur Incorporation.

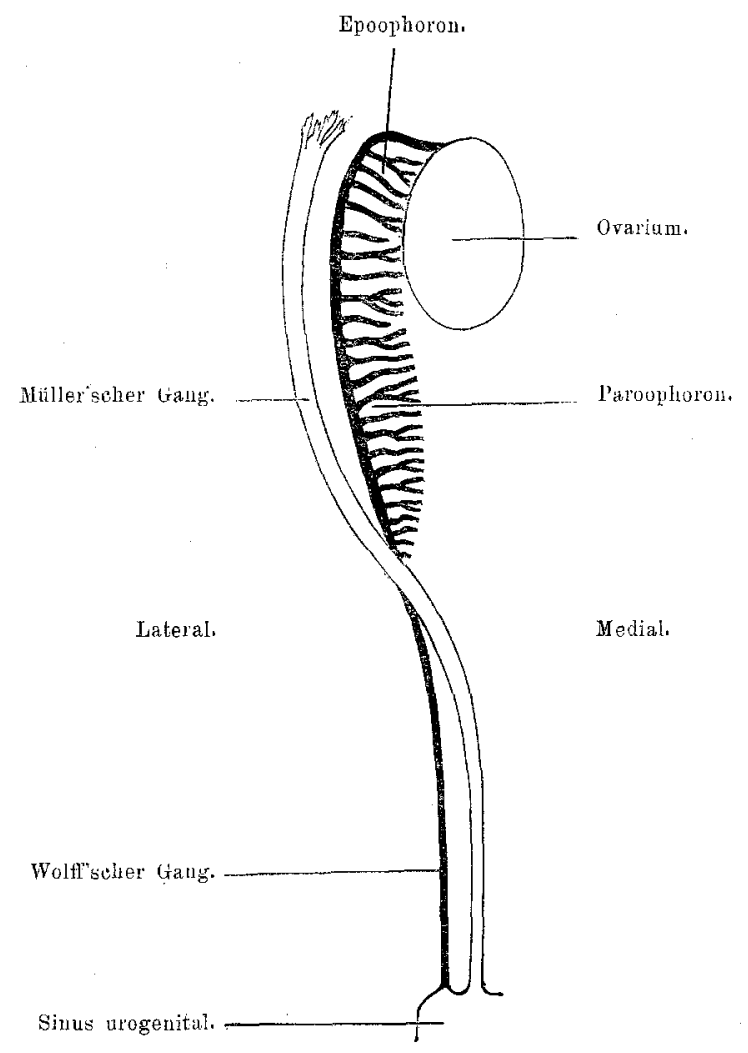

Die mehrfach erwähnte Umbiegungsstelle der Urnierenfalte, an der die Kreuzung vom Wolff'schen Gang und Müller'sehen Gang

1) Die enge nachbarliche Beziehung zwischen paroophoralen Umierencanälchen und Müller'schem Gang ist übrigens auch aus der nach Walde yer ausgeführten Zeichnung Hertwig's (15, Fig. 198, S. 294) ohne Woiteres zu erkennen.

2) Ich erwähne z. B. den Befund A. Czerny's, der (v. Recklinghausen, 1, S. 127) bei einem einjährigen Knaben deutliche Glomeruli im mittleren Drittel des Samenstranges fand. 
stattfindet, ist nun bekanntlich die Stelle des Tubenwinkels. Sio wird schon früh äusserlich durch den Abgang des Leistenbandes der Unniere markirt (s. z. B. Nagel, 20, S. 71). Bis hierher schreitet später die Vereinigung der Plicae urogenitales - oder genaucr der Müller'schen Fäden — zu Scheide und Uterus von unten nach oben hin fort; der proximalwärts davon gelegene $\mathrm{Ab}$ schnitt des Müller'schen Ganges liefert den Eileiter.

Danach ergiebt sich ohne Schwierigkeit, dass dic Incorporation von distalen (paroophoralen) Urmierencanälchen in die Substanz der Müller'schen Gänge, mag sie nun einen regelmässigen Vorgang oder eine "Missbildung per excessum" (v. Recklinghausen, 1, S. 209) darstellen, sich vollziehen muss am Rücken des künftigen Gebärmutterkörpers und zwar insbesondere in der Gegend des Kreuzungspunktes mit dem Wolffschen Gang: auf der dorsalen Seite der Tubenwinkel und des Uterusscheitels; ferner dass sie wesentlich und zunächst die peripherischen Schichten des Organs betreffen muss. Genau an diesen Stellen des Uterus aber liegen, wie v. Recklinghausen (1, S. 151 und S. 158) hervorhebt, zugleich auch die Prädilectionsstellen seiner Adenomyome, und genau an diesen Stellen werden, wie z. B. unsere Beobachtung (2) zeigt, die hierher gehörigen Adenomyome wiedergefunden.

Auch alle weiteren Eigenschaften der v. Recklinghausenschen Adenomyome: die Symmetrie der Tubenwinkeltumoren, die Beschränkung der T'ubenadenomyome auf die mediale Eileiterhälfte und das Freisein der Cervix (physiologisch stärkste Atrophie am distalen Urnierentheil!) passen genau zum Ablauf des geschilderten Inclusionsprocesses, ja, lassen sich als directe Folgerungen aus diesem ableiten.

Dabei setzt natürlich diese Anschauung, was ich besonders betonen möchte, nicht bloss die "Incorporation" von Urnierentheilchen in den Müller'schen Gang voraus, sondern auch zugleich die Eigenschaft dieser aus ihrem natürlichen Verband gelösten Gewebsbestandtheile, der physiologisehen. Atrophie zu widerstehen und früher oder später in selbständiger Wucherung sich zu entfalten.

Jedenfalls muss man keineswegs, wie v. Lockstädt (4, S. 37) meint, nach v. Recklinghausen erwarten, auf Adenomyome am häufigsten in der abdominalen Hälfte der Tube zu stossen oder sie an der Tube viel häufiger als an der Gebärmutter zu treffen. Genau das Gegentheil ist richtig. 
Scheidengewölbes, ihre Stellung zu den paroophor. Adenomyomen etc. 469

Ebenso ist die Voraussetzung Kossmann's für seinen Gegenbeweis, dass (3, S. 374, S. 376) der Wolff'sehe Gang stets Müller'schen Gang, bezüglich Myometrium, und Urnierencanälchen trennt und so ihren Einschluss in die Corpusmusculatur hindert, nur bedingt anzuerkennen, d. h. sie trifft (s. das obige Schema) allein für den proximalen Abschnitt des Müller'schen Ganges, also für die Wand des Eileiters zu, in dessen lateralem Abschnitt bisher Adenomyome auch in der That niemals gefunden worden sind (v. Lockstädt, 4, S. 21, L. Pick, 2, S. 173.

Nach der Kreuzung liegt der Müller'sche Gang; d. h. das Myometrium des Uterus genau so an der medialen Seite des Wolff'schen Ganges wie die Urnierencanälehen, und wenn Kossmann ans den Quersehnitten seines Embryons schliesst (4, S. 374), dass "die letzten Canälchen der Urniere (u) relativ weit $(270 \mu)$ cntfernt vom Uterus" liegen, so ist dieser Schluss ein irrthümlicher. Denn die in Fig. 8 abgebildete verschmolzene Partie der Müllerschen Gänge ist nicht der ganze künftige Uterus, sondern nur der unterste Theil derselben. Die Versehmelzung der M. G. zum Uterus schreitet nach oben hin viel weiter bis zur ursprünglichen Kreuzungsstelle des Müller'schen und Wolff'schen Ganges, also noch über Fig. $7 \mathrm{zu}$ Fig. 6 hin, fort. Die Lagebeziehung der letzten Urnierencanälchen zu diesem Kreuzungspunkte, das ist's, worauf es ausschliesslich -- auch für künftige Untersuchungen - ankommt.

Zuzugeben ist, dass die Lehre $\nabla$. Recklinghausen's unter Berücksichtigung des neven Materials gewisser Correcturen und Erweiterungen bedarf.

1. Zunächst ist die Trenunug von paroophoralen und schleimhäutigen Adenomyomen des Uterus als peripherischen und centralen (v. R., 1, S. 233 und S. 234) höchstens im Allgemeinen aufrecht zu erhalten.

Dass Ausläufer paroophoraler „peripherischer" Adenomyome sich innenwärts an die Utertsschleimhaut annähern können und ferner die teleangiectatischen paroophoralen Adenomyomformen in den inneren Schichten des Myometrium corporis anzutreffen sind, hat bereits v. Recklinghausen (1, S. 232, S. 93 u.) selbst gelehrt. Andererseits steht nun aber jetzt fest, dass schleimhäutige Adenomyome sich durchaus nicht nur auf die inneren Lagen der Uterus- oder etwa aussehliesslich der Ventralwand des Uterus (v. R., 1, S. 231) beschränken. Im Fall VII v. Lockstädt's z. B. (4, S. 13-15), 
wo cin diffuses Adenomyom in ganzer Oberfächenbreite mit der Schleimhaut zusammenhängt und in das Stratum submucosum und vasculosum des gesammten Myometriums eingelagert ist, besteht dic Entwicklungshöhe in der Dorsalseite und im Fundus (S. 25). Ebenso in Fall V (4, S. 8-9). Das centrale schleimhäutige Adenomyom in Fall VII dringt mit Ausläufern nicht nur bis zur Serosa und in die Tubenecken hinein, sondern steht durch einen directen Fortsatz mit einem kirschgrossen subserösen Fundusadenomyom am uterinen Ende der rechten Tube in ummittelbarem Zusammenhang $(4$, S. 15, S. 25$)$.

Es ist nicht allein die innige embryonale Verbindung des Wolff'schen und Müller'schen Ganges oder gar neine Art Missbildung" (v. Recklinghausen, 1, S. 167), die diese gelegentlich schr auffällige Ineinanderschiebung der beiderseitigen Gangderivato crklärt. Vielmehr ist hier direct an den embryonalen Incorporationsprocess als solchen anzuknüpfen. Werden Urnierencanälehen von der Substanz des Müller'schen Ganges umschlossen, so sind sie keineswegs wie etwa der Aufdruck eines Stempels an dieser Stelle fixirt. Mag es der Lymphstrom sein, der die abgesprengten Theilchen weiterführt ${ }^{1}$, mögen diese mit den Blutgefässverzweigungen sich verbreiten oder mögen sie an den rein mechanischen Wachsthumsverschiebungen aller Gewebe theilnehmen - man erimnere sich der auf diese Weise erfolgenden Verlagerung abgelöster Nebennierentheile z. B. in die Leber -- jedenfalls sind sie an die Dorsalwand und die peripherischen Schichten der Gebärmutter nicht deswegen absolut gebunden, weil sie hier aufgenommen wurden. Sie sind hier ein-, aber nicht abgeschlossen.

So stossen wir auf centrale oder auch ventral gelegene „paroophorale" Adenomyome, wenn eben auch nur als Ausnahme.

1) Riess (Eine neue Operationsmethode des Uteruscarcinoms, Zeitsohı. f. Geburtsh. u. Gynäkol., Bd. 37, S. 518, 1897) fand bei einem typischen Plattenepithelkrebs der Cervix und Metastasen des Carcinoms in den iliacalen Drüsen in zweien derselben, in ihrer Substanz wie im periglandulären Bindegewebe, drüsenähnliche Bildungen mit ausgeprägtem Cylinderepithel; ebenso in einem Stück des linken Ligamentum sacfo-uterinum; ausserdem Adenomyome beider Tubenwinkel. R. folgert, wie mir seheint, ganz richtig (S. 526), "dass bei der Entwicklung der Genitalien ein Theil des Wolff' schen Körpers sich an und in diese Lymphknoten verirrt hat,", und zwar möchte ich annehmen, dass sowohl in diese Lymphdrüsen, wie wohl auch in das linke Ligam. sacro-uterinum der Transport incorporiter Theile des W. K. mit dem Lymphstrom erfolgte. 
Auf der anderen Seite sind embryonal abgesprengte und verlagerte Theile der Müller'schen Gänge (R. Meyer, 13a) und ihre Derivate auch in den äusseren Schichten des späteren Myomotriums anzutreffen.

Offene Fragen bleiben vorläufig noch, inwieweit postfötale Wucherungen des Endometriums die Fähigkeit besitzen, im Myometrium sich centrifugal auszubreiten (vergl. v. R., 1, S. 234), wieweit also angeborene Anlagen und postfötale Proliferation in die Erzeugung der schleimhäutigen Adenomyome sich theilen; ferncr inwieweit mit Combinationen schleimhäutiger und paroophoraler Adenomyome gerechnet werden muss (vergl. z. B. v. Recklinghausen, Fall II, 1, S. 232).

2. Den Adenomyomgruppen am Genitalschlauch, die v. Recklinghausen umschreibt (1, S. 4), gliedern sich neue charakteristische Abtheilungen an.

So ist durch v. Jockstädt (Fall VII, 4, S. 15 und S. 28) immerhin wahrscheinlich gemacht, dass auch rom Tubenepithel her Adenomyomschläuche geliefert werden können, also auch am Eileiter schleimhäutige, seien es embryonal angelegte oder postfötal gebildete, und paroophorale Adenomyome zu trennen sind.

Einen weiteren anatomisch wohlumschriebenen Typus bilden, wie ich zeigen konnte (2), die voluminösen subserösen und iuxtauterinen Adenomyome. Die practische Bedeutung gerade dieser Gruppe ist eine erhebliche. Denn während in allen Fällen voluminöser intraparietaler Gebärmutteradenomyome bei der Ausschneidung der Geschwülste wegen ihrer diffusen Abgrenzung gegen das Myometrium stets der Uterus geopfert werden muss (Freund, 1a, S. 225), gelingt hier die isolirte Exstirpation bez. die Enucleation der Geschwïlste (2, S. 164). Zu dieser Gruppe sind ausser unserem Tumor, ihrem Begründer, die neuerdings veröffentlichten Fällo Gebhard's (11, S. 430), Pfannenstiel's (7, S. 197), v. Lockstädt's Fall IV und VI (4, S. 8--10), vielleicht auch die beiden subserösen Adenomyome in v. L.'s Fall V (4, S. 11), zu rechnen, soweit die Beschreibungen eine sichere Deutung gestatten. Bei Gebhard, Pfannenstiel und in v. Lockstädt's Fall VI wurde, wie den Geschwülsten dieser Gruppe eigenthümlich, conservativ verfahren, in v. Lockstädt's Fall IV und VI der Uterus aus anderer Indication exstirpirt.

Endlich giebt es typische grössere oder kleinere Kugelmyome, die einzelne drüsige Gänge oder Cystchen oder selbst einige adeno- 
matöse Inselcheu enthalten (v. Recklinghausen, Fall I, III, IVb, 1, S. 185; Orloff, 12, 4 Fälle; v. Lockstädt, Fall I, II, III, 4, S. 6-8; R. Meyer, 13a, S. 334). Im Gegensatz zu den „infiltrirten" Adenomyomen stellen diese Tumoren stets wohlumgrenzte leicht enucleirbare Geschwülste oder Gesehwülstehen dar.

Es dürfte sich hier um den zufälligen, rein passiven Einschluss embryonaler epithelialer Ueberbleibsel in Kugelmyome handeln, um so mehr dann, wenn gleichzeitig echte wohlausgebildete Adenomyome bestehen (1, Fall I, III, IVb). Können doch auch umschriebene Kugelmyome von diffusem Adenomyom eingeschlossen werden $(4$, S. 13, S. 400 .).

Weniger bedoutungsvoll unter den neuen Errungenschaften der Adenomyomlehre sind etliche weitere Hypothesen für die Genese der epithelialen Schläuche und Cysten: Abkunft von embryonal verlagertem Ovarial- bez. Keimepithel (4, S. 31, 34, 39), von versprengten Elementen des Gartner'schen Ganges (eod. loc.) oder vom Peritonealepithel (Iwanoff 1 ). Für diese Theorien liegt um so weniger ein Bedürfniss vor, als der doppelte Ursprung der Schläuche und Cysten der Adenomyome aus dem Epithel des Müller'schen Ganges oder ron den Urnierencanälchen gesichert erscheint und für die Erklärung aller Fälle zwanglos genügt.

Die typische Localisation eines Adenomyoms am Uterusdorsum bez. Fundusdorsum und die specifische Urnierenform seiner Epithelschläuche: das sind ausreichende Beweismittel für seinen „paroophoralen" Ursprung ${ }^{2}$ ).

Man darf nur nicht vergessen, dass die eindeutigen und wirklich beweisenden Urnierenformationen wesentlich in den drüsigen "Inseln" und "Guirlanden", den "geschlossenen Ordnungen" der voluminösen intraparietalen Corpusadenomyome getroffen werden und dass solche Fälle unter der Zahl der relativ bäufigen Adenomyome offenbar recht selten sind. v. Recklinghausen's erste $(1$, S. 3,

1) Iwanoff, Zur Frage über die Adenomyome des Uterus. Inaug.-Diss. 1897. (Russ.)

2) Die Bedeutung dieser Thatsachen im Sinne der Urnierentheorie erkennt (s. o. S. 462) auch r. Lockstaedt an. Gegenüber v. L.'s Kritik meiner Befunde (4, S. 49), will ich nur betonen, dass wie v. Recklinghausen, so auch ich selbst eine Abtheilung der Canälchen in gewundene Secretionsröhrchen und gestreckte Sammelröhrchen gefunden (2, S. 165), ja, abgebildet habe (Taf. II, Fig. 9), wenn ich freilich auch nicht, so wenig wie v. R., an jedem Röhrchon diese Differenzirung feststellen konnte (1, S. 104 r..). 
$96,104)$ und unsere Beobachtung (2) sind, wie schon Eingangs hervorgehoben, bisher die einzigen dieser Art.

Meiner Ansicht nach sind diese Fälle schon genügend, gegenüber Kossmann und $\mathrm{v}$. Lockstaedt das Vorkommen wirklicher paroophoraler Adenomyome im Sinne v. Recklinghausen's darzuthun.

Immerhin aber hielt ich es nach den Angriffen dieser Autoren für zweckmässig, das Gebäude der neuen Lehre durch weitere Stützen zu festigen.

Denn es scheint an und für sich gewiss wünschenswerth, bei der mehr und mehr erkannten Häufigkeit der Adenomyome am Gẻnitalschlauch ihre Histogenese möglichst zu klären.

Lässt sich zumal die Existenz der paroophoralen Adenomyome v. Recklinghausen's wirklich überzeugend sichern, so ist damit eine ausserordentlich bemerkenswerthe Geschwulstkategorie geschaffen.

Wie unter den histioiden Tumoren das Myxom ein embryonales Gewebe reproducirt, das sich im Körper des Erwachsenen höchstens in spärlichen Resten findet, so zeigt unter den organoiden Geschwülsten das paroophorale Adenomyom gelegentlich in voller Ausbildung die complicirte Drüsenstructur des Wolff'schen Körpers wieder, eines embryonalen Organs, das im breiten Mutterband des erwachsenen. Weibes nur durch unbedeutende Ueberreste vertreten wird. Die Geschwulstbildung, dort die histioide, hier die organoide, bedeutet einen Rückschlag in das embryonale Leben, ein Wiederauftauchen dort eines längst entschwundenen Gewebes, hier eines eigenartigen, fast völlig verlorenen Organs.

Nun ist freilich ein typisches Adenomyom als unmittelbare Abzweigung eines Gartner'schen Ganges (vgl. v. Reeklinghausen, 1, S. 102) oder das Vorkommen paroophoraler specifischer Urnierenabschnitte im tumorfreien Uterus- ader Tubenfleisch ${ }^{1}$ ) bisher nicht

1) Die unter diesem Titel veröffentlichten Befunde (Ricker, 13, Paroophoron im Uterus; Rieder, 24, S. 115 u., Ueberreste des Wolff'schen Körpers [Glomeruli und Canälchen der Umiere] zu beiden Seiten am Rande des Myometrium corporis; v. Recklinghausen, 1, S. 161 u. 162, Urnierencanälchen im tumorfreien Tumorwinlel: Fall VI, XXIV, XXV, und im Uterushorn der Kuh; L. Pick, 2, Gruppe von Wolff'schen Canälchen in der isthmischen Hälfte einer Doppeltubo) zeigen, unbeschadet der Richtigkeit ihrer Deutung; keine specifischen Strukturanalogien mit dem Wolff'schen Körper. 
sicher erwiesen. Diese unstreitig zwingenden directen Argumente für v. Recklinghausen's Theorie fehlen vor der Hand noch.

Vielmehr kann eben der Beweis für die paroophorale Abstammung der Adenomyome - wie ja auch der für die schleimhäutige Abkunft aller. Adenomyome im Sinne Kossmann's und v. Lockstädt's - nur indirect, durcb Indicien, erbracht werden. So mussten zu den Gründen v. Recklinghausen's für seine Anschauung neue möglichst sichere und beweiskräftige "Indieien" gesucht und hinzugefügt werden.

Wo in der Eileiter- oder Uteruswand oder auch unmittelbar neben dem Uterus, im Parametrium, Adenomyome und Cystadenome sitzen, ist es, man mag über v. Recklinghausen's Theorie so günstig denken wie man nur will, sicherlich unmöglich, a limine die Annahme abzulehnen, dass die drüsigen und eystischen Formen dieser Tumoren vom Epithel der Müller'schen Gänge herstammen könnten. Wolff'seher Körper und Gang auf der einen, Müller'scher Gang auf der anderen Seite stehen in einem so innigen embryonalen Connex und, da der Müller'sche Gang bekanntlich physiologisch den Wolff'schen auf einer grossen. Strecke umschliesst, auch in so enger postfötaler Verbindung, dass dem anatomischen Situs nach für die Epithelien der Adenomyome und Cystadenome der Uterus- und Tubenwandung von vornherein gewiss beide Ursprungsmöglichkeiten zur Erörterung stehen. Und dies um so mehr, als, wie schon Eingangs hervorgehoben, viele Adenomyome des Uterus mehr oder weniger cytogenes Bindégewebe enthaiten, das im Endometrium corporis stets, in Umierenresten bisher noch niemals nachgewiesen ist $\mathbf{1}$.

Darum stelle ich die Frage zunächst so:

1. Giebt es in Verbindung mit dem weiblichen Genitalschlauch, ausserhalb von Tube und Uterus, Adenomyome und Cystadenome, die ohne besondere Rücksicht auf ibre specifischen histologischen Eigenschaften schon allein durch ihre Position ausschliesslich auf den Wolff'schen Körper als Ausgangspunkt hinweisen?

2. Wenn Geschwülste dieser Art und Abstammung vorkommen, führen auch diese cytogenes Gewebe?

In der That giebt es an den weiblichen Genitalien Cyst-.

1) Hervorzuheben scheint mir der Nachweis cytogenen Bindegewobes in der Schleimhaut des cervicalen Abschnittes des Gartner." schen Ganges bei v. Recklinghausen (1, S. 70, Fall 19). 
adenomyome, für deren Epithel trotz ihres Bestandes an cytogenem Gewebe die Abstammung vom Wolff'schen Körper die einzig mögliche Annahme bildet.

Stimmen aber diese zweifellosen Derivate der Uruiere mit den Drüsenbestandtheilen der Cystadenomyome der Uterus- und Tubenwand in Anordnung und Structur überein, so liegt darin unter Berücksichtigung der oben (S. 464-468) ausführlich geschilderten embryonalen Lageverhältnisse des Wolff'schen und Müller'schen Ganges ein positiver Beweis dafür, dass auch in Tube und Uterus Geschwülste dieser Kategorie gleichfalls von Urnierenresten entspringen können, zumal wenn ihr Sitz sich in vollendeter Weise mit den Forderungen dieser Annahme deckt oder gelegentlich gar directe Analogieen der adenomatösen Partieen mit Urnierenformen zu sehen sind. Die Hypothese der Entwicklung der Epithelelemente in Uterus- und Tubenadenomyomen aus dem Epithel der Müller'schen Gänge wäre nicht a priori die allein wahrscheinliche oder auch nur wahrscheinlichere.

Cystadenomyome, die nach diesen Gesichtspunkten als sichere Beweismittel der paroophoralen Theorie v. Recklinghausen's dienen, sind in der Leistengegend und im hinteren Scheidengewölbe zu finden.

Ein günstiger Zufall hat mir die Untersuchung je eines typischen Exemplars dieser noch wenig gekannten Tumoren ermöglicht. Meine Präparate vom Adenomyom der Leistengegend entstammen dem von Frl. Dr. Agnes Bluhm jüngst beschriebenen Fall (8). Frl. Bl. nahm Veranlassung, mir ihre makro- und mikroskopischen Befunde zu demonstriren und überliess der Sammlung unserer Klinik. in dankenswerther Weise eine Anzahl von Sehnitten der Geschwulst. Der Fall von Adenomyom der Scheide, der mir besonders lehrreiche Präparate bescheerte, wurde von Herrn Dr. Theodor Landau der Klinik überwiesen und operirt.

Weitere Beschreibungen von Scheidenadenomyomen rühren von v. Herff (9) und Pfannenstiel (7) her; in Pf.'s Fall fand sich zugleich ein Adenomyom der Leistengegend. Sonst haben Tumoren letzterer Art nur noch v. Recklinghausen (5) und Cullen (6) beschrieben.

Im Falle Bluhm (8) war bei einer 20 jährigen Nullipara in der rechten Jeistengegend eine über pflaumengrosse schmerzhafte Geschwulst herangewachsen. Bei der Exstirpation erwies sie sich in das letzte Stück des Ligamentum rotundum eingesehaltet; das in 
den Tumor unmittelbar eintrat. Medianwärts gingen aus letaterem strahlenförmig derbe Bindewebsbündel hervor, die sich in der Gegend des Mons veneris ansetzten. Eine circumscripte Auslösung der Geschwulst war nieht möglich.

Der makroskopische Durchschnitt des Präparates zeigt "zahlreiche theils kugelige, theils schlauchförmige, stecknadelkopf- bis kirschkerngrosse Hohlräume, die zum grösseren Theil miteinander communiciren und einen blutig tingirten serösen Inhalt haben. Die Septa sind mässig derb". Mikroskopisch (vergl. 8, Taf. XXVIT, $1,2,3)$ setzt sich das Geschwulststroma aus fibrillärem kernarmen Bindegewebe und kleineren Zügen glatter Muskelfasern zusammen. Starker Gefässreichthum, stellenweise perivasculäre Rundzellenanhäufungen. Die am makroskopischen Durchschnitt sichtbaren polymorphen Cysten stellen sich als ein endothelausgekleidetes Netzwerk vielfach gebuchteter, engerer oder weiterer anastomosirender Canäle, also als zweifellose Lymphangiektasieen dar.

Neben diesen findet sich bei weitem spärlicher eine zweite Art wohlcharakterisirter Hohlräume, die auf den ersten Blick als Drüsenimponiren. Sie erscheinen meist isolirt, zuweilen auch in Gruppen und tragen ein schönes pallisadenförmiges nicht deutlich flimmerndes Cylinderepithel. Von der durchsichtigen Grundsubstanz des Tumors sind sie allerwärts durch breitere oder schmälere Lagen oines Stromas geschieden, das stellenweise durchaus der Tunica propria der Uterusschleimhaut gleicht. Gewisse Drüsenquerschnitte sind halbmondförmig, indem hier das cytogene. Bindegewebe das Lumen concav einbaucht. Auf dieser concaven Lumenseite ist das Epithel höher als auf der convexen.

Sowohl in dem rundzellreichen periglandulären Stroma, wie auch besonders in vielfachen Rundzellanhäufungen, die von anssen knotenförmig in die Lymphangiektasieen vorspringen, trifft man Capillaren, freie rothe Blutkörperchen und gelbliches bis tief rostbraunes Pigment; theils frei, theils in grossen Zellen eingeschlossen.

Die drüsigen Hohlräume treten in den Bluhm'schen Präparaten gegenüber den lymphangiektatischen Cysten und Gängen ziemlich zurück. Immerhin möchte ich die Geschwulst bei der zweifellosen besonderen Diguität dieser epithelialen Gebilde als ein lymphangiektatisches Adenomyofibrom des Jigamentum rotundum bezeichnen.

Einer erheblicheren Entwicklung des Drüsenapparates begegnen wir in dem Falle Cullen's (6). Hier wird bei einer 34jährigen Ipara. 
aus dem oberen Theil des rechten grossen Labjum ein Geschwulstknoten $(7: 4: 3,5 \mathrm{~cm})$ sammt der bedeckenden Haut herausgeschnitten. Der Tumor hatte der Trägerin schneidende Schmerzen verursacht, die nach dem Rücken hin ausstrahlten und nach körperlicher Anstrengung oder zur Zeit der Periode exacerbirten. Er stand, wie die Operation ergab, in unmittelbarer Verbindung mit dem runden Mutterband. Dieses schloss auf seiner Verlaufsstrecke zwischen innerem und äusserem Muttermund einen zweiten Knoten (1,6 cm Durchmesser) ein, der mitsammt dem Ligament gleichfalls excidirt, indess später nicht untersucht wurde.

Der makroskopische Geschwulstquerschnitt lässt unter normaler Haut in stark hämorrhagischem Fettgewebe einen aussergewöhnlich festen Knoten $(3,5: 3: 2 \mathrm{~cm})$ von etwas diffuser Begrenzung erkennen. Fig. 1 (6) demonstrirt dies Lageverhältniss in natürlicher Grösse. Er zeigt ein dichtes fasriges Geflecht mit vielen kleinen unregelmässigen blassen homogenen und transparenten Einsprengungen, in denen nach der Härtung runde, ovale oder irreguläro Hohlräumchen auftauchen. In den mikroskopischen Präparaten bunt durchflochtene, glatte Muskelfasern, vielfach ein gelbbraunes, körniges Pigment einschliessend. Strotzende Gefässfüllung.

In. allen Richtungen wird diese Grundmasse von längs- und quergetroffenen, gelegentlich dichotomisch verzweigten Drüsen durchzogen, die nebst ihrem umgebenden Stroma (Fig. 2) von der Uteruskörperschleimhant nicht zu unterscheiden sind und sowohl isolirt wie in grossen Complexen angetroffen werden. Manche Drüsenräume sind gross, weit, halbmondförmig. Cytogenes Gewebe füllt ihre Concavität und schliesst zahlreiche Capillaren, Hämorrhagien und einen oder mehrere drüsige Schläuche ein, deren Einmündung in den grossen Hohlraum geiegentlich festzustellen ist. Diesem zellreichen Stroma sitzen cylindrische Flimmerepithelien auf, während an der Convexität die Epithelien meist flach und vom musculären Stroma nicht weiter getrennt sind. In dem grossen halbmondförmigen Lumen lagern rothe Blutkörperchen, abgestossene und degenerirte Epithelien, theilweis mit braunen Pigmentkörnchen gefüllt. Auch das Epithel der übrigen Drüsenbildungen ist, wo es der musculösen Grundsubstanz direct auflagẹt, ganz gesetzmässig cubisch oder flach, wo Stroma sich dazwischenschiebt, cylindrisch. Nur einige wenige kleine Drüsen lagern unmittelbar zwischen den Muskelbündeln. 
Cullen beschreibt den Tumor als "Adenomyoma of the round ligament".

Von der Beobachtung v. Recklinghausen's ist nur soviel berichtet (5), dass die Geschwulst, aus der Leistenbeuge einer Frau. entfernt, ein Leiomyom darstellte, das drüsenartige epithelausgekleidete Schläuche enthielt und wohl als ein Adenomyom des. Tigamentum rotundum zu deuten war.

In Pfannenstiel's Fall (7) - Combination von Adenomyon der Leistengegend und Scheide - wurden bei der 39jährigen Patientin wegen erheblicher Beschwerden die chronisch entzündeten rechten Anhänge, ein Geschwulstknoten aus der Regio inguinalis dextra und, da auch im hinteren Scheidengewölbe und im Uterus je ein knotiger Tumor zu fühlen war (verschiedene Metastasen einer bösartigen Neubildung?), Gebärmutter sammt hinterem Fornix exstirpirt. Wie die vaginale Totalexstirpation, so war auch die Ausschneidung des Leistentumors recht mühsam, weil dieser ganz diffus mit den aus dem Leistencanal ausstrahlenden Gewebspartien verschmolzen war. Dabei sass die Geschwulst von kaum Walnussgrösse etwas nach innen und unten vom äusseren Leistenring, ohne nachweisbare Verbindung mit dem Ligamentum rotundum, das bej der Operation gar nicht zu Gesicht kam. Das hintere Scheidengewölbe war in einen kleinen dem Uterus nahe anliegenden Tumor umgewandelt und zeigte auf dem Durchschnitt, ebenso wie die Geschwulst der Leistengegend, kleine gelblich braune Pigmentflecken und feine Spalten und Cysten, auf der sonst glatten Schleimhautseite einige kleine cystische Vorbucklungen. Mikroskopisch trifft man in beiden Geschwülsten drüsenschlauchähnliche Massen und Pigmentkörperchen, überhaupt "die typische Structur des Adenomyoms" v. Recklinghausen's (7, S. 197 u.). Der Uterusknoten erwies sich als ein kleines Myom.

v. Herff hat seine Mittheilung „über Cystomyome und Adenomyome der Scheide" (y) durch Ito in dessen Dissertation "über Fibrome, Cystofibrome und Adenomyofibrome der Scheide" (9a) ergänzen lassen.

Bei einer 49 jährigen 4 para bestand Prolapsus uteri et vaginae, starke Hyperplasie der.Portio, die trocknen. Epithelüberzug und cin Decubitalgeschwür aufwies.

An der Absatzstelle der Portio gegen die Scheidenwand, ca. $1 / 2 \mathrm{~cm}$ von der hinteren Muttermundslippe entfernt, sitzen auf der hinteren Vaginalwand 2 polypöse Tumoren ziemlich dicht neben- 
einander (9a, Fig. 2). Der linke kleinere, von fast Walnussgrösse, zeigt einen $2 \mathrm{~cm}$ langen, fast fingerdicken Stiel. Der rechte grössere sitzt mit einem dicken breiteren Sticl auf. Beide sind von glatter Scheidenwand überkleidet und von mässig fester Consistenz. Klinische Diagnose: Fibromata pendula fornicis raginae.

Bei der mit vorderer Kolporrhaphie verbundenen vaginalen Totalexstirpation (Prof. Fehling) wird der circuläre Schnitt um die Portio so geführt, dass die hintere Scheidenwand, die die Basis der oben erwähnten Fibrome bildet, mitumschnitten wird.

Beide Polypen bestehen aus lockerem zelligen Bindegewebe mit Zügen glatter Muskelfasern, die in verschiedenen Richtungen verlaufen. Unter dem Deckepithel, schon für das blosse Auge sichtbar, liegen zahlreiche selbst 3--4 mm im Durchmesser haltende Hohlräıme, die allerwärts von kubischem oder cylindrischem, stellenweise stark abgeplattetem Epithel ausgekleidet sind, durch eine "Membrana propria" begrenzt werden und zelligen Detritus und Schleim enthalten. Gelegentlich "Andeutungen von Flimmerbelag.". Daneben ächte schlauchförmige leicht geschlängelte, stellenweise 3-4 mm lange Drüsen, in denen hohes Cylinderepithel mit basalen Kernen der Membrana propria aufsitzt. Auch hier scheinen Flimmern aufzutreten. Den sehr engen Ausführungsgang kleiden zerfallende, anscheinend leicht verhornte Plattenepithelzellen aus, die sich einerseits in das Plattenepithel der Oberfläche, andererseits in die Cylinderepithelschicht der Drüsen fortsetzen. Die Cysten unter der Epithelschicht sind, wie Reihenschnitte lehren, zweifellos aus diesen Drüsen hervorgegangen: sie sind Retentionscysten derselben. Beide Tumoren sind also polypöse Fibromyome, die Retentionscysten ächter Scheidendrüsen einschliessen: Cystofibromyomata pendula.

Ausserdem aber finden sich, in der Tiefe der einen Geschwulst" (9a, S. 11 u.) zahlreiche feinste Drüsenröhrchen in inselartiger dichter Gruppirung, von einer schmalen Schicht begrenzter glatter Muskulatur umgeben und von vereinzelten kleinen runden und oblongen Cysten flankirt. Drüsenepithel allerwärts einschichtig, kubisch oder niedrig cylindrisch, ohne Bildung von Becherzellen oder sonstiger Secretionsformen. Hicr und da sind die Röhrehen mit Erweiterungen oder Seitenästen versehen, an einer Stelle in sternartiger Anordnung. Die kleinen Cysten messen nur wenige Zehntel Millimeter und besitzen niedriges Cylinderepithel, gleichfalls ohne Zeichen einer Schleimsecretion. Einzelne der 
Drüsenröhrchen münden in Cystchen. Flimmern nirgends nachweisbar.

Eins der Cystomyofibrome ist also zugleich noch ein Adenofibromyom.

Schliesslich unser eigener Fall:

Frau E. J., 31 J. Aufgenommen 28. April 1898. 3 para. 0 ab. Stets regelmässig menstruirt, seit $1 \mathrm{Jahr}$ wegen Unterleibsbeschwerden in poliklinischer Behandlung. Vor $1 / 2$ Jahr wurde ein haselnussgrosser Tumor im hinteren Scheidengewölbe bemerkt, der sich seitdem stetig vergrössert hat, so dass der Pat. die Excision der (malignen?) Neubildung empfohlen wird. - Grosse kräftige Frau. Scheide von mittlerer Weite, Portio kurz, breit. Uterus von normaler Grösse, in Anteflexionsstellung. Im hinteren Scheidengewölbe ist eine pflaumengrosse Geschwulst zu fühlen. Sie ist sehr resistent und hart, wenig beweglich, von intacter Scheidenschleimhaut überzogen, die sich über ihr nicht verschieben lässt.

In Narkose wird die Portio angehakt und die Geschwulstoberflache umschnitten. Unter stumpfem Vorpräpariren in die Tiefe wird der Tumor mit ziemlicher Mühe ausgelöst und mitsammt der deckenden Scheidenschleimhaut excidirt. In der Tiefe der Wunde kommt das an einer kleinen Stelle eröffnete Bauchfell des Douglas in Sicht. Es wird mit einigen Catgutsuturen vereinigt, die Wundhöhle mit Gaze drainirt. Glatter Verlauf. Pat. genesen entlassen. Andauerndes Wohlbefinden.

Die ausgeschnittene derbe Geschwulst besitzt die Dimensionen $23: 15: 15 \mathrm{~mm}$ und ist an der Oberfläche von glatter Scheidenschleimhaut ïberzogen. Hier und da haften Reste (paravaginalen) Fettgewebes an. Ein Durchschnitt auf der Höhe des Tumors zeigt eine grauliche leicht gefaserte Grundsubstanz, in der namentlich in der Tiefe der Geschwulst bis $1 \mathrm{~mm}$ breite weissliche Züge in Form einer netzförmigen Aederung sich verbreiten. Ziemlich zahlreich sind in unregelmässiger Vertheilung strich- und fleckförmige bräunliche rothbraune oder orangegelbe Pigmentirungen eingesprengt. In den netzförmigen Adern, die an den Knotenpunkten sich nur unbedeutend verdicken, tauchen besonders deutlich bei Lupenvergrösserung sehr häufig feine punkt- und spaltförmige, oft gekrümmte Canälchen auf. Die weissliche Scheidenschleimhant ist gegen das im Ganzen mehr graue Tumorgewebe scharf abgesetzt, von wechselnder Dicke $(11 / 2-3 \mathrm{~mm})$ und bietet an mehreren Stellen ein eigenthümliches Verhalten dadurch, dass ziemlich tief im Schleinhanstroma, dem Geschwulstgewebe genähert, einige linsengrosse scharf umschriebene, peripheriseh bräunliche und im Centrum hellere Körper gelegen sind, die gleichfalls feine Spalten enthalten und, wie weitere Querschnitte lehren, mit der Schleimhautoberfläche continuirlich verbunden sind.

Zur mikroskopischen Untersuchung gelangen Schnitte durch die ganze Dicke der exstirpirten Masse (Celloidineinbettung, Färbung mit Alaunkarmin und Haemalaum, Eosin\}.

An der Oberfläche erscheint die Scheidenschleimbaut, deren Epithel stellenweise nur in Resten (mechanische Schädigung bei der Scheidendesinfection ante operationem) erhalten ist, stellenweise sich aber in normaler Weise schichtet. Das Schleimhautstroma ist kaum verdickt, enthält aber zahlreiche dilatirte leere oder blutgefüllte Capillaren, denen sich gelegentlich Infiltrationsherdchen anschliessen. Da und dort auch 
einzelne oder gruppenförmig gehäufte kleinere und grössere braungelbe Pigmentkörnchen und -kügelchen.

Die mehrfach in die Schleimhaut eingelagerten makroskopischen bräunlichen Körperchen zeigen einen auffallenden Bau: das geschichtete Oberflächenepithel senkt sich bier mit sämmtlichen Lagen kryptenförmig ein und formirt unter continuirlicher Verschmälerung schliesslich einfache Schläuche, die sich mit einiger Verästelung terminal unregelmässig erweitern. Ziemlich kräftiges Cylinderepithel in einfacher Lage bildet die Schlauchwand. Der erweiterte Endabschnitt schliesst körnige Gerinnungsmasse und zelligen Detritus ein. Das Ganze ist zunächst von einer dichten Rundzellenanhäufung umkapselt, die namentlich um die Tubuli herum ron prall gefïllten Capillaren durchzogen wird. Die äusserste Schale der kugeligen Körperchen bilden mehr minder umfängliche Hämorrhagieen, durch welche auch die makroskopisch bräunliche Färbung der Peripherie bedingt wird. Hin und wieder finden sich auch zwischen den Rundzellen Pigmentkörner. Ueberall sind diese Körperchen in ihrer Ausdehnung ausschliesslich auf das Schleimhautstroma beschränkt und von der darunterliegenden Scheidenmuskelhaut noch durch reichliches Bindegewebe getrennt.

An den Randpartien der exstirpirten Masse liegt unter der etwas verdickten Scheidenmuskelschicht das lockere paravaginale, reichlich Fettzellen einschliessende Bindegewebe. Schnell nimmt dann die Muskelhaut an Dicke zu und schwillt unmittelbar zu der kugligen eigentlichen Geschwulst an. d. h. diese tritt als directer Auswuchs aus der Scheidenmuskelschicht in's paravaginale Zell-(Fett)-gewebe heraus. Nach der Scheidenseite hin ist die Grenze zwischen Muskel- und Schleimhautschicht glatt erhalten. An der nicht ganz regelmässigen, mehr welligen und etwas diffusen Grenzlinie der Tumormasse gegen das paravaginale Zellgewebe reichliche Blutungen, zahlreiche weite Gefässchen und leukocytäre Infiltrationsherdchen.

Als unmittelbarer "Auswuchs" der Scheidenmusculatur ist die Geschwulst um so mehr zu bezeichnen, als das typisch fibromusculäre Geschwultsstroma mit seiner Structur in das Scheidenmuskelrohr durchaus continuirlich übergeht: man sieht hier wie dort schmälere und breitere Fascikel glatter Muskelfasern, von durchsichtigen Bindegewebsstrassen getrennt. Der Gefässgehalt ist nicht auffallend.

Daneben zeigt nun die fibromusculäre Grundmasse des Tumors einen ausserordentlichen Reichthum an drüsigen Bildungen.

Die feinen weisslichen Aederungen, welche sich auf dem Geschwulstdurchschnitt makroskopisch heraushoben und schon makroskopisch deutliche Spältchen einschlossen, stecken voll von dicht gehäuften glandulären Formen. Andererseits sind auch zahlreiche isolirte Drüsenbildungen in das Fibromyom eingesprengt. Erstere sind mehr nach der Tiefe, letztere mehr nach der Oberfläche der Geschwulst, schleimhautwärts, gelagert.

In den weisslichen Adern umschliesst typisch cytogenes Stroma die dicht gelagerten Schläuche, die entweder wesentlich auf dem Querschnitt oder Längsschnitt getroffen, d. h. im Ganzen parallel gelagert sind. In bogigem oder mehr gestrecktem Verlanf setzen sich die Adern mit einander in viclfache Verbindung, so dass sich die netzförmige Durchflechtung des Fibromyoms mit einem aus cytogener Grundmasse und drüsigen Einschlüssen bestehenden System leicht demonstriren lässt.

Die Schläuche auf den Verbindungsstrecken zwischen den Knoten- 
punkten (s. Fig. 2, $a_{1} a_{2} a_{3}$, Fig. $3, s_{2} s_{3}$ ) sind eng; gleich kalibrirt, windungslos, sanft gebogen und wegen ihres parallelen Verlaufs oft auf Quer- oder Schrägschnitten einzeln hintereinander („im Gänsemarsch") aufgestellt. Besonders auffallend ist an einzelnen longitudinal getroffenen Röhrchen, die überhaupt nicht selten auf sehr langen Verlaufsstrecken zuweilen mit scharfer bogiger Umbiegung zu verfolgen sind, eine wie an den Zweigen eines Baumes mehrfach hintereinander auftretende dichotomische Verästelung (Fig. $1, \mathrm{~h}$ und $\mathrm{d}_{1} \mathrm{~d}_{2}$ ).

Eine Anschwellung der Adern an den Vereinigungspunkten zu umfänglichen "Inseln" besteht, entsprechend dem makroskopischen Verhalten, nur in unbedeutendem Maasse. Diese Vereinigungsknoten haben insgesammt eine im Ganzen mehr langgezogene Form; die Adern vereinigen sich in ihnen nicht unter sternförmigem Eintritt, sondern setzen sich astförmig an verschiedenen Stellen seitlich an (Fig. 2, $\mathrm{a}_{1} \mathrm{a}_{2} \mathrm{a}_{3}$ ). Dabei beruhen die unerheblichen Verbreiterungen an den Vereinigungsstellen nicht sowohl auf einer Zunahme der eintretenden Schläuche oder des cytogenen Gewebes, als vielmebr auf Dilatationen der eintretenden Drüsenröhrchen. Das enge gestreckte Kaliber weitet sich auf Kosten des gelegentlich stark rareficirten cytogenen Stromas zu meist ganz unregelmässigen zackigen, langgezogenen Räumen aus (Fig. 2, $\mathrm{r}_{1} \mathrm{r}_{2}$ ) oder es treten auch mehrere dünne Schläuche zu diesen zusammen.

In der Regel betrifft diese sinuöse Erweiterung sämmtliche Epithelröhren einer solchen Insel. Indem das Epithel, cytogenem Gewebe aufsitzend, in grösseren und kleineren, mehroderminder zahlreichen papillären. Erhebungen, die selbst wieder mit Aus- und Einstülpungen versehen sein können, in das Lumen der ausgeweiteten Räume hineinragt, sind in diesen nicht selten quer und schräg getroffene Papillen allerverschiedenster Form und Zahl mit cytogenem Grundstock und einschichtigem Epithelsaum anzutreffen (Fig. 2, $\mathrm{r}_{2}$ ).

Gelegentlich sieht man in den InseIn nur einen einzigen ganz besonders grossen erweiterten Epithelraum, dam meist von Halbmondform, dessen Boden durch das cytogene Stroma, oft unter reichlicher Papillenbildung eingestülpt wird und dessen Dach, nach aussen convex gewölbt, direct dem Fibromyomgewebe aufsitzt. Auf günstigen Schnitten lässt sich zu g:leicher Zeit die Einmündung der engen Schläuche in den Boden dieser Ampulle, die Abzweigung bogiger Adem ("Inselschenkel") aus der Insel und der Verlauf von Röbren von einer Insel des Systems zur nächsten mit Einmündung von Schläuchen in einen ampullären Raum derselben, nachweisen. An einer Stelle münden drei parallel ziehende langgestreckte Röhren in einer Ebene nebeneinander in einen quergestellten weiten Canal, wie die Zähne eines Kammes in dessen Rückenleiste.

Ferner besteht, wie an den engen Röhren, auch an einigen der weiten grossen Canäle der Inseln (auf Längsschnitten sichtbar!) eine ausgesprochene Dichotomie, und an diese Verästelungen des weiten Rohres setzen sich unter neuer Abzweigung wieder enge, event. noch weiter getheilte Drüsenschläuche als Nebenzweige an (Fig. 3): das Bild eines grösseren Sammelrohrs ( $h$ ), das unter fortgesetzter gabliger Theilung von allerwärts her Aeste und Aestchen $\left(s_{1} s_{2} s_{3} s_{4}\right)$ aufnimmt. An diesen Systemen sowahl wie an anderen Stellen beginnen die engen Sehläuche mit einer kugligen oder olivenförmigen oder mehr unregelmässigen (Fig. 3, 0 und $\left.\mathrm{u}_{1} \mathrm{u}_{2}\right)$, zuweilen in einer anderen Insel gelegenen Auftreibung, an die sich gelegentlich auch erst noch eine kurze geschlängelte Strecke 
anschliesst, oder es wölbt sich in das rund aufgebanchte Endstück eines Rohrs ein kugliger Knopf aus cytogenem Bindegewebe und einem Ueberzug kubischen Epithels.

Je mehr man aus der Tiefe der Geschwulst gegen die Schleimhaut vorrückt, desto melır verliert sich der geschlossene Zusammenhang der Drüisenformen und das cytogene Gewebe, desto mehr treten isolirte Formen auf und desto grösser ist die Neigung dieser zerstreuten Ausläufer zur cystösen Erweiterung.

Ziemlich häufig werden halbmondförmige zweizipflig ausgezogene relativ weite Epithelräume gefunden, die den erweiterten Haupträumen in den Inseln gleichen; dann auch mit dem gesetzmässigen Aufbau, dass der eventuell papillenbesetzte Boden gehäuftem cytogenen Gewebe, das Dach dem Fibromyomgewebe - zuweilen auch noch Spuren des ersteren - auflagert. Oder aber man trifft mehr unregelmüssige, nicht einfach runde Cystchen, die von einer faser- und spindelzellreichen Hülle oder unmittelbar vom Fibromyom umkapselt sind. Doch fehlt im Ganzen cytogenes Gewebe in Begleitung der Epithelformationen selten völlig.

Von diesen versprengten epithelialen Formen zu den dichten geschlossenen Verbänden nach der Tiefe der Geschwulst bin finden sich unter entsprechender Vermehrung der cytogenen Grundmasse alle Uebergänge, rudimentäre Inseln oder Verbindungsstücke etc., deren Schilderung im Einzelnen sich erubrigt. Durchaus vermisst werden, so gross auch die numerische Hypertrophie der Drüsen sei und so dicht diese auch stehen mögen, die bekannten Korkzieher- und Doppelsägeformen, wie sie die Corpusdrüsen der Gebärmutter so häufig bei chronisch entzündlicher Wucherung zeigen; ferner irgendwie erhebliche Cystenbildungen von makroskopischem Umfange.

Bei der Abgrenzung des cytogenen Gewebes gegen das Fibromyomgewebe bin ich Schwierigkeiten nicht begegnet; zuweilen schiebt sich zwischen beide eine Zone spindelzell- und fibrillenreicherer Bindesubstanz, in das cytogene Bindegewebe allmählich übergehend, aber vom Muske]gewebe scharf abgesetzt.

Irgendwelche Beziehung der Muskelbündel zu den zerstrenten odor geschlossenen Ordnungen der Drïsenformen in Form von "Scheiden" ist, trotz der allerwärts ausgesprochenen Streckung der drïsigen Verbände, nirgends dentlich.

Das Epithel sämmtlicher drüsiger und cystischer Formen ist einschichtig. Gelegentlich ist eine von scharf tingirten flachen Spindelzellen gebildete Tunica propria zu sehen.

Die Elemente der engkalibrirten Schläuche sind ausnahmslos cylindrisch mit durchsichtigem Plasma und ovalen, dunkelgefärbten Kernen, die in den basalen Zellhälften in etwas ungleicher Höhe nebeneinandergereiht sind. Ein Kernkörperchen ist zuweilen deutlich. - Auch in den erweiterten Röhren der Inseln hält sich das Epithel im Allgemeinen hoch. Nur wo das cytogene Bindegewebe als Unterlage schwindet, also an dem convexen Dach der halbmondförmigen in den Inseln gelegenen oder isolirten Räume plattet sich das Epithellager regelmässig stark $a b$, so dass dann eine augenfällige Differenz zwischen diesem und dem hocheylindrischen Zellbelag des mehr oder weniger papillären Bodens besteht. - Die im Muskelgewebe isolirt versprengten Cysten führen in der ganzen Circumferenz platte Epithelien, und endlich tritt unbeschadet der cytogenen Lnterlage ein platteres Epithel da auf, wo die engen Drüsenröhren in weiteren Anschwellungen endigen. 
Flimmern sind mit voller Deutlichkeit nirgends nachzuweisen; ebensowenig Becherzellen oder sonstige Secretionsformen.

Ziemlich häufig sind Blutungen im cytogenen Bindegewebe am Boden der isolirt stehenden Ampullen, zumal in den papillären Bodenerhebungen; hier sind dann gewöhnlich auch die Ampullen selbst mehr oder weniger mit frischem Blut gefüllt. SpärJicher sind Hämorrhagieen im Inselstroma oder in den weiteren oder engen Röhrchen der Inseln anzutreffen. Dabei werden gefüllte Blutgefässe im cytogenen Gewebe eigentlich nirgends ganz deutlich sichtbar. Um so auffalliger ist es, dass an einzelnen Stellen die drüsigen Inseln um eine kleine oder selbst mehrere relativ dicke Arterien als Centrum sich gruppiren, von diesen geradezu durehbohrt werden oder sich in unmittelbarer Nachbarschaft seitlich an sie anlagern, während sonst in dem fibromyomatösen Grundgewebe kleine Gefässe dieses Kalibers durchaus nicht häufig sind.

Hier und da liegt bräunliches Pigment in Form polymorpher Körner; eventuell neben Blutkörperchen in der cytogenen Grundmasse der Inseln; gelegentlich stösst man dort auch auf umschriebene Häufchen auffallend grosser runder, mit grossen Kernen versehener Elemente, die mit feinsten gelbbraunen Granulis rollgestopft sind.

Die Hauptmasse des. Pigments liegt im interstitiellen Bindegewebe des fibromyomatösen Geschwulstantheils. Ohne bestimmte engere Beziehnng zu den Drüsen- und Cystchenformen, wenn auch oft in naher Nachbarschaft derselben, ist es wie diese in allen Tumortheilen anzutreffen; gelegentlich auch, ebenso wie reine Hämorrhagien, in besonderer Nähe von Blutgefässen. Grössere und kleinere gelbbraune oder mehr braune Partikel, mehr oder weniger dicht gelagert, intra- oder extracellulär, verbreiten sich in diffuser; unregelmässiger Weise mit der intermusculären Bindesubstanz und schaaren sich selbst zu makroskopisch deutlichen Herdchen. Eisenreaction am Pigment nirgends deutlich.

Ausser reinem Blut enthalten Ampullen oder Cystchen gelegentlich auch abgestossene Epithelien, Leukocyten, grosse Pigmentzellen, pigmentirte hyaline Kugeln, farblose hyaline Massen oder amorphen körnigen Detritus in verschiedener Combination.

Mehrere Male fand ich die Endauftreibungen der engen Drüsenröhrchen weit gedehnt durch kuglige Agglomerate glänzender concentrisch geschichteter Concretionen. Aehnliche Körper von mehr drusiger Zusammensetzung lagern auch frei im Fibromyomgewebe.

Schliesslich habe ich noch einen eigenartigen Befund aus den Rindenschichten der Geschwulst zu erwähnen: von einer kräftigen Pigmentzone und circulär streichenden Muskelzügen umrahmt sind grosse knötchenförmige Körper anzutreffen, die sich aus Pigmentzellen, amorphem Pigment, Leukocyten mit runden und fragmentirten Kernen, zarten epitheloiden Elementen und feinen Capiliaren in buntem Durcheinander aufbauen.

Da in einzelnen dieser Herde noch Lumina resp. Epithelmassen in charakteristischer Anordnung auftauchen, so erweisen sich die auffallenden Körper als in Organisation begriffene hämorrhagisch infarcirte drüsige Tnseln.

Diese genauen Ausführungen genügt es vorläufig kurz dahin zusammenzufassen: Der vorliegende Tumor ist eine Mischgeschwulst. Ein kugliges Fibromyom wölbt sich im hinteren Fornix raginae 
aus dem Muskelrohr der Scheide ins paravaginale Zellgewebe knotig vor. Es führt namentlich in der Tiefe reichliche Einlagerungen echter Drüsen, also adenomatöse Bestandtheile, ist mithin ein A de no m yom.

Stellt man die bisher bekannten Fälle von Adenomyomen der Leistengegend (v. Recklinghausen [5], Cullen [6], BIuhm [8], Pfannenstiel [7]) und der Scheide (Pfannenstiel [7], v. Herff [9]) nebeneinander, so erkennt man eine Anzahl gemeinsamer Eigenschaften, die weit über die in all' diesen Tumoren gleichartige Zusammensetzung aus fibromusculärem und drüsigem Gewebe hinausgehen.

Es handelt sich um fast walnuss- bis pflaumengrosse harte Geschwülste, die sich zwar eimigermassen scharf gegen ihre Umgebung absetzen, aber doch diffus genug in diese ausstrahlen, um ihre isolirte Entfernung zu erschweren.

Die einen sitzen in der Leistengegend (3mal rechts, 1 mal ?), cntweder ganz in der Nähe (medial) vom äusseren Leistenring oder schon im oberen Theil der Schamlippe. Sie sind theils dem Ende des runden Mutterbandes eingesehlossen, gelegentlich sogar, wie es scheint, in mehrfachen Herden ${ }^{1}$ ), theils ist eine Verbindung mit diesem nicht nachweisbar. Sie sind somit generell am besten nicht als Adenomyome des runden Mutterbandes, sondern als Adenomyome der Jeistengegend zu bezeichnen.

Die anderen sitzen übereinstimmend im hinteren Fornix; alle bisher bekannt gewordenen vaginalen Adenomyome sind Adenomyome des hinteren Scheidengewölbes. Sie springen entweder in continuo aus der Substanz des Fornixmuskelrohrs in das paravaginale Zell- resp. Fettgewebe bis nahe zum Uterus vor oder stielen sich polypös in das Scheidenlumen hinein. Die überdeckende Scheidenschleimhaut ist intact, glatt, kann aber dabei kleine cystische Vorbucklungen zeigen.

Die Adenomyome der Leistengegend und des hinteren Fornix können sich combiniren (Pfannenstiel, 7).

Auf dem makroskopischen Quersehnitt präsentirt sich mehr oder weniger deutlich die bekannte grauröthliche desmoide Zeichnung des Fibromyoms. In dieser aber fallen eventuell kleine gelbe oder braune Pigmentfleckchen, sowie oft feine regelmässige oder irregu-

1) Falls nämlich die zweite Geschwulst, welche sich bei Cullen (6) im Ligamentum rotundum zwischen innerem Leistenring und dem Adenomyom am äusseren Ende des Ligamentum rotundum fand, aber nicht untersucht wurde, auch ein Adenomyom war. 
läre Spalten und Cystchen auf'. Zuweilen stecken diese in besonderen weisslichen ramificirten oder fleckförmigen Einsprengungen.

Die Grund- und Hauptmasse der Tumoren besteht, wie das Mikroskop bestätigt, aus regellos angeordnetem Fibromyomgewebe, bei zuweilen nur geringem museulösen Antheil. Im Fall Bluhm (8) enthält die myofibromatöse Grundsubstanz reichliche, selbst cystische Lymphangiektasien.

Steht das einkapselnde Gewebe mit zur Untersuchung, so markirt sich unter Umständen die nicht absolut scharfe Trennung desselben von der Geschwulstmasse dadureh, dass in dieser einzelne Herdchen z. B. des subcutanen (Cullen) oder paravaginalen Fettgewebes auftauchen oder doch mindestens die Grenzlinie der Tumorsubstanz gegen die Umgebung mehr diffus unregelmässig; wellig verläuft.

Die epithelialen Einschlüsse treten entweder in grösseren Schaaren als wesentlicher Tumorbestand oder in spärlicherer Quantität mehr vereinzelt auf. Durchgreifend ist der Unterschied indessen nicht. Auch im ersten Falle stösst man stets auf einige mehr isolirte Ausläufer. Diese isolirten Formen zeigen sehr gewöhnlich Neigung zu cystischer Ausweitung in Gestalt halbmondförmiger Ampullen.

Wo die Epithelformationen sich in Schaaren (Inseln und Strängen oder Adern) zusammenschliessen, sind sie stets von reichlichem cytogenen Bindegewebe eingescheidet. So kommen die makroskopischen weisslichen, Spältchen führenden Geäste und Flecken zu Stande. Das cytogene Bindegewebe fehit auch keineswegs in der Umgebung vereinzelt stehender Drüsen oder Cystchen, - es findet sich namentlich unter dem nach innen convexen Boden der halbmondförmigen Ampullen -, doch führen die isolirten Epithelformationen oft mehr kernarme fasrige Scheiden oder stecken gelegentlich direct im Fibromyomgewebe.

Das Epithel ist stets einschichtig, zuweilen flimmernd, ohne deutliche Secretionsformen (Becherzellen). Die Grundform in den einfachen Schläuchen bildet das Cylinderepithel. Wo es cytogenem Bindegewebe aufsitzt, erfolgt kaum je - abgesehen vielleicht ron den Kolbigen Endausweitungen mancher enger Röhren - bedeutendere Abflachung. Auf fasrigem Bindegewebe oder Muskelgewebe trifft man dagegen gesetzmässig kubische oder platte Formen. 
Hämorrhagien und Pigmentmassen, frei oder in grossen Zellen ("Pigmentkörper"), sind im capillarreichen cytogenen Stroma oder innerhalb der Epithelräume, in den Drüsenschläuchen wic namentlich in den Cysten und Ampullen, zu finden. Auch die eventuell reich vascularisirte fibromyomatöse Grundsubstanz zeigt hämorrhagische Herde und Pigmentansammlungen im interfasciculären Bindegewebe oder auch in den vergrösserten Muskelelementen.

Sonst trifft man in den Drüsen und Cysten noch Leukocyten, abgestossene und veränderte Epithelien, zelligen Detritus, hyaline pigmentirte oder farblose Massen, auch lrystallinische Concretionen.

Die hämorrhagische Zertrümmerung ganzer drüsiger Inseln führt zur Bildung eigenthümlicher knötchenförmiger, pigmentführender und von Pigmentschalen unkapselter Herde, die von der Umgebung aus organisirt werden. Vor einer Verwechselung derselben mit echten Tuberkeln schützt - von Anderem (Pigmentirung, Zellformen) abgesehen - vor Allem ihre Vàscularisation.

Bei den drei Adenomyomen des hinteren Schejdengewölbes sind zweimal - bei v. Herff (9) und in unserem Fall - zugleich Scheidendrüsen nebst - bei v. Herff - zahlreichen Retentionscystchen derselben beobachtet. Letztere, unmittelbar unter der Epithelschicht gelegen, messen bis $3-\mathbf{4} \mathrm{mm}$ im Durchmesser.

Es ist wahrseheinlich, dass Scheidendrüsen sich auch mit dem fornicalen Adenomyom im Fall Pfannenstiel (7) combiniren. Denn auf der sonst glatten Schleimhaut über der Geschwulst treten "kleine cystische Vorbucklungen" heraus.

Um hier auch gleich die klinischen Daten zu erledigen, so ist aus dem bisher vorhandenen Material Folgendes abzuleiten:

Die Tumoren sind ausschliesslich bei geschlechtsreifen Frauen noch vor der Klimax (im Alter von 20, 31, 37, 39, 49 Jahren) gefunden. Das Wachsthum ist meist ein überaus langsames und zieht sich nicht selten durch eine Reihe von Jahren hin.

Die Adenomyomknoten in der Leistengegend können stechende nach dem Rücken ausstrahlende Schmerzen erzeugen, die sowohl bei der Periode als auch bei irgendwelchen körperlichen Anstrengungen solche Heftigkeit erreichen, dass sie die Arbeitsfähigkeit herabsetzen und zur Exstirpation des Tumors drängen.

Die Fornixadenomyome bedingen in den bisher beobachteten bescheideneren Dimensionen weniger subjective Symptome, als dass sie bei der eigenartigen diffusen Beschaffenheit der knotigen Einlagerung den Gedanken an eine maligne Neubildung aufkommen Iassen, zumal wenn, wie bei Pfaunenstiel, gleichzeitig noch ein Tumor der Leistenbenge (vermeintliche Drüsenmetastase) und im Uterus besteht. Sie 
sind zwar von glatter Schleimhant überzogen, das ist aber z. B. bei der knotigen submucösen Form des Scheidensarkoms auch der Fall ${ }^{1}$. Ihr anscheinend typischer Sitz im hinteren Fornix vaginae besitzt indessen wohl eine gewisse differentialdiagnostische Bedeutung.

Die Adenomyome der Leistengegend scheinen bei der klinischen Untersuchung gelegentlich durch einen Strang (Ligam. rotund. uteri) mit dem Leistencanal in Verbindung zu stehen. Sie fordern zur Differentialdiagnose mit Lymphdrüsenschwellungen, Hydrocele muliebris, Inguinalund insbesondere Ovarialhernien auf, weil sie, wie übrigens auch die Hydrocele muliebris oder Tumoren des Ligamentum rotundum anderer Art, menstruelle Anschwellung und Vergrösserung zeigen können. Mehr als im besten Falle eine solide Geschwulst des Ligamentum rotundum wird allerdings kaum jemals herauszudiagnosticiren sein.

Die Geschwülste sind durchaus gutartig. Seitdem durch Rolly (14) jedoch die krebsige Metamorphose echter paroophoraler Adenomyome sichergestellt ist ${ }^{2}$, bleibt immerhin zu erwägen, ob nicht gewisse genetisch dunkle Carcinome des Septum recto-vaginale aus krebsiger Umwandlung paroophoraler Adenomyome am Scheidendorsum hervorgehen mögen.

Unmittelbar aus dem anatomischen Befund ergiebt sich, dass die fibromyomatösen Bestandtheile der Adenomyome der Leistengegend der Muskelsubstanz des runden Mutterbandes entstammen, selbst wenn der Zusammenhang des Tumors mit dem an seinem inguinalen Ende bekanntlich oft stark aufgefaserten und rareficirten Band nicht oder, genauer, nicht mehr ausgesprochen ist, und ferner, dass das Fibromyomgewebe der fornicalen Adenomyome von der Muskelhülle des Scheidengewölbes geliefert wird.

Was aber ist die Matrix der im Tumorgewebe eingeschlossenen drüsigen und eystischen Epithelformen?

Was zunächst diejenigen der Adenomyome der Leistenbeuge betrifft, so haben v. Recklinghausen, Pfannenstiel und Bluhm sie von embryonal versehleppten Urnierencanälchen, Cullen von embryonal versprengten Theilen des Müller'schen Ganges hergeleitet ${ }^{3}$ ).

1) Vergl. L. Pick, Ueber Sarkome des Uterus und der Vagina im Kindesalter und das primäre Scheidensarkom der Erwachsenen. Dieses Archiv. Bd. 46. 1894. S. 217.

2) Vergl. auch Iwan off, Drüsiges cystenhaltiges Uterusfibromyom, complicirt durch Sarkom und Carcinom. Monatsschr. f. Geburtsh. u. Gynäkol. Bd. 7. H. 3. März 1898. S. 219.

3) v. Loclistaedt denkt daran (4, S. 37), für die Adenomyome der Leistenbenge r. Recklinghausen's und Cullen's, die Epithelien "aus den daselbst normalerweise vorkommenden Bartholini'schen Drüsen" entstehen zu lassen. Ein curioser Eiror loci! 
Für ihre genetische Aufklärung muss man an dasjenige Entwicklungsstadium des weiblichen Urogenitalapparates anknüpfen, in welchem - im dritten Monat des Fötallebens - ein bandartiger, glatte Muskelfasern einschliessender ununterbrochener Strang rom unteren Pol des Wolff'schen Körpers zur Leistengegend zieht: das Leistenband der Urniere. Es sondert sich, entsprechend seinen Beziehungen zum Wolff'schen Körper, dem Eierstock und dem Müller'schen Gang, in drei continuirlich verbundene Abschnitte. Der erste zieht vom Wolff'schen Körper (Parovarium) zum Hilus ovarii, der zweite von dort zum Tubenwinkel (Lig. ovar. inferius), der dritte von hier zur Leistengegend (Lig. inguinale + Conus inguinalis). Das Ligamentum ovarii inferius wird später zu dem kurzen straffen Strang des Jigamentum o varii proprium, das Ligamentum inguinale + Conus inguinalis zum Ligamenten uteri rotundum ${ }^{1}$ ). Diese Thatsachen sind bei zehnwöchentlichen Embryonen zuerst ron Wieger (16) gefunden ${ }^{2}$ ) und ganz neuerdings von M. Blumborg und B. Heymann (18) für den Menschen und des Weiteren auch für die Säugethiere gegenüber Klaatsch (19) bestätigt.

Die Verbindung des Urnierenleistenbandes mit dem Müllerschen Gang ist nun - das ist hier der springende Punkt -- eine rein äusserliche. Der "continuirliche Zug" des Ligamentum ovarii inferius (ovarii proprium) und inguinale (rotundum) heftet sich auf der Dorsalseite der Tube in Gestalt eines mit der Lupe distinet sichtbaren "Wulstes" aussen an.

Diese Verhältnisse lassen sich nun noch weiter in sehr frühe Entwicklungsstufen zurückverfolgen: Blumberg und Heymann fanden das Ligamentum inguinale und ovarii inferius in den gcschilderten Verhältnissen zum Müller'schen Gang resp. zum Tubenwinkel gleichmässig an 5 menschlichen Embryonen aus dem zweiten Monat (18, S. 282). D. h. also: das Ligamentum uteri teres steht bej seiner Anlage im zweiten Monat durch unmittelbare proximale Fortsetzung mit dem unteren Pol des um diese Zeit drüsig noch reich verzweigten Wolff'schen Körpers in innigster Verbindung, während es dem Müller'schen Gang, der auf dieser Entwicklungs-

1) Das Ligam. uteri rotundum ist nicht gleichwerthig (Hertwig, 15, S. 287; Nagel, 20, S. 72 u. 81) mit dem Gubernaculum Hunteri des Mannes. Letzteres, vom Kopf des Nebenhodens zur Symphyse ziehend, entspricht der ganzen Länge des Urnierenleistenbandes, ersteres nur dem distalen, allerdings wesentlichsten Theil.

2) Vergl. auch v. Milıálkovicz (17). 
stufe nur ein einfaches glattes Epithelrohr einschliesst, an seiner mesodermalen Aussenschicht wulstförmig, rein äusserlich auflagert.

Diese Thatsachen, auf die zum Theil A. Bluhm bereits zutreffend hingewiesen hat, gestatten nur den einen Schluss, dass sei es bei der ersten Anlage oder gelegentlich der beim Descensus ovariorum besonders ausgiebigen Wachsthumsverschiebungen Theile des untersten Unierenabschnittes, Paroophoron- oder diesen adaequate Canälchen in das Urnierenleistenband und weiterhin in seinen distalen Abschnitt, das Ligamentum inguinale resp. den Conus inguinalis, hineingolangen und mit diesem durch die Bauchwand hindurch in die Umgebung des äusseren Leistenrings, zum Mons pubis oder in den oberen Theil der grossen Labie. Das Ligamentum inguinale übernimmt für die paroophoralen Einschlüsse, wie für das Ovarium, die Rolle des "Leitbandes".

Somit sind die epithelialen Einschlüsse der Adenomyomeder Leistengegend Abkömmlingevonverschleppten distalen i. e. paroophoralen Uruierencanälohen ${ }^{\mathbf{1}}$ ).

Diese Ableitung ist gegenüber der vom Epithel der Müller'schen Gänge hier so wahrscheinlich, dass sogar Kossmann (3, S. 373) für die Drüsen des Cullen'schen Falles eher geneigt ist, auf den Wolff'sehen als auf den Müller'schen Gang zurückzugreifen ${ }^{2}$ ).

Die Drüsen und Cystchen der Adenomyome des hinteren Scheidengewölbes bezieht Pfannenstiel in seinem Fall auf verlagertes Paroophoron, das netwas abnorm tief bis zur. Scheide

1) Es liegt darin ein Hinweis, auch im normalen Ligamentum rotundum nach paroophoralen Epitheleinschlüssen zu suchen. In Gemeinschaft mit Herrn Dr. Henry L. Williams-Philadelphia habe ich eine Anzahs runder Mutterbänder von Neugeborenen im Laboratorium unserer Klinik systematisch auf vollkommenen Schnittserien untersucht, indessen vor der Hand mit negativem Erfolg.

2) A. Bluhm (18) hat auch für die Tubenwinkeladenomyome einen ähnlichen Entstehungsmodus - embryonalen Transport von Urnierencanälchen nach dieser Stelle hin durch das Urnierenleistenband -- angedeutet.

Obschon gelegentlich gewiss nicht bloss am Eude, sondern auch im Verlauf des runden Mutterbandes Aclenomyome auf diese Art entstehen mögen, erklärt sich Entwicklung und Häufigkeit der Tubenwinkeladenomyome doch́ ungezwungen und überzeugend genng, wie oben ausführlich dargestellt, aus der directen Incorporation von Urnierencanälchen in den Müller' schen Gang. bei der physiologischen Kreuzung des letzteren mit dem. Wolff' schen Gang bezw. Körper. 
hinabragte". v. Herff und Ito (9, S. 190, 192 und 9 a, S. 23) lassen die Möglichkeit offen, sie von abnormerweise erhaltenen caudalen Abschnitten des Wolff'schen Körpers oder den "wurzelförmigen Ausbuchtungen" abzuleiten, die der Wolff'sche Gang mit Vorliebe in der Cervix uteri treibe und die sich hier einmal ausnahmsweise in der Höhe des Scheidenansatzes statt wie sonst im Cervixgewebo entwickelt hätten.

Jedenfalls aber stellen $v$. Herff und Ito ihr fornicales Adenomyom den paroophoralen Tumoren v. Recklinghausen's ausdrücklich zur Seite.

Die "wurzelförmigen" oder "wurzelstockartigen" Anhänge der Wolff'schen Gänge im Uterushals spielen auch bei anderen Autoren in der Adenomyomfrage eine gewisse Rolle. So bei v. Recklinghausen selbst, der wie v. Herff und Ito die von der cervicalen "drüsigen Einrichtung " der Gartn er'schen Gänge gelieferten Epithelschläuche und Cysten (1, Fall 17 und Fall 21) mit den Adenocysten seiner paroophoralen Adenomyome in Parallele rückt (1, S. 154, 181) oder bei Klein, der generell die Adenomyome von Recklinghausen's von verzweigten Resten des Wolff'schen Ganges ableitet, die etwa im Uterus persistiren (10, S. 166).

"Drüsige" oder "traubenförmige" Anhängsel, Ausstülpungen und Verzweigungen am Gartner'schen Gange sind sowohl bei Thieren (Schwein, Kalb) wie beim Menschen ${ }^{1}$ ) in der Cervix uteri, gelegentlich selbst noch im oberen Theil der Vagina (z. B. von Gartner beim Schwein, von Rieder bei einem 14jährigen Mädchen) ${ }^{2}$ ) gefunden. "Gegen die Mitte der Cervix", so sagt Rieder (S. 125) bei der Beschreibung des Verlaufes der Gartner'schen Gänge beim Menschen, ,erscheinen regelmässig (sc. wo der Gang überhaupt vorhanden ist) Verästelungen und Ausstülpungen, wie man sie sonst nirgends an denselben beobachtet. Man findet gowöhnlich ein längliches Lumen und zu beiden Seiten massenhafte ganz mit Epithel ausgekleidete Schläuche, so dass oft ein Lumen in denselben fehlt." Sie rücken mit ihren Endausläufern bis dicht an die blinden Enden der Cervixdrüsen (v. Recklinghausen, 1, S. 38, Fall 7, und S. 160; Rieder, 10, S. 115, Fall 1; unsere Fig. 4, Tat. XXIII).

1) Vergl. 7. B. bei Rieder (24) die Befunde Gartner's, Follin's, Rienor's bezw. Dohrn's, Rieder's; ferner Klein (10, S. 448).

2) Vergl. Rieder (10, S. 101 u. 115). 
v. Recklinghausen sah sie zweimal in Combination mit Adenomyomen des Uteruskörpers (1, Fall 7 und Fall 19).

Abbildungen dieser "drüsigen Einrichtung" in der Cervix geben u. A. Rieder und Dobrn (25) für die menschliche Gebärmutter, Rieder auch für den Kalbsuterus.

Ich selbst zeige in Fig. 4 auf Tafel XXIII cin typisches Beispiel derselben (G. G. $+\mathrm{dr}$ A). Der Mutterhalsquerschnitt eines ausgetragenen todtgeborenen Mädchens zeigt das bekannte w förmige Lumen. Das hocheylindrische durehsichtige Schleimhautepithel ist nur an wenigen Stellen der Schleimhautoberfläche, besser in den ziemlich tiefliegenden Drüsenquerschnitten (Dr) enthalten.

Mitten in die circulär streichende Musculatur $(\mathrm{m})$ der rechten Cervixseite eingebettet sieht man in Gestalt einer scharf umschriebenen Insel den Gartner'schen Gang nebst seinem Drüsenanhang. Nach vorn zu treibt der Gang (G. G.) eine kurze schlauchförmige Abzweigung. Seine Epithelien sind, wie stärkere Vergrösserung lebrt, nur an einzelnen kleinen Stellen noch erhalten - der Schnitt ist von uneingebettetem Material angefertigt -, die der drüsigen Abzweigungen dagegen allerwärts vortrefflich conservirt. Sie sind einschichtig, kubisch, führen einen dunkelgefärbten, rundlichen, die Zelle fast völlig ausfüllenden Kern mit Kernkörperchen und sind so von den langen, schmalen, hochcylindrischen durchsichtigen Cervixdrüsenepithelien, die wie beim Erwachsenen ausgeprägt basalständige Zellkerne einschliessen, auf das Markanteste verschieden. Diese Epitheldifferenz ist auch auf der Figur mit Leichtigkeit sichtbar. Das Stroma der Insel besteht aus faserigem, sehr spindelzellreichen Bindegewebe. Als ein eigentlich cytogenes Gewebe kain man es nicht bezeichnen.

Wären wirklich dic Epithelien dor paroophoralen Uterusadenomyome v. Recklinghausen's von diesen drüsigen intrauterinen Epithelverzweigungen der Gartner'schen Gängo abzuleiten (Klein) oder wären auch nur die Producte der letzteren denen paroophoraler Unierenabschnitte gleichzustellen (v. Recklinghausen, v. Herff, Ito), dann wären ja die gesuchton epithelialen Grundlageu der Adenomyome im Myometrium gefunden. Zum mindesten dürfte dann mit Sicherheit geschlossen werden, dass zwișchen dicsen dem Paroophoron äquivalent gesetzten Schläuchen im Cervixfleisch und dem Paroophoron Waldeyer's, im medialen Liga- 
mentum latum, weitere paroophorale Abschnitte im Myometrium corporis erhalten sein müssen.

Dass dieser Schluss und damit auch seine Voraussetzung unzutreffend ist, erhellt eigentlich schon zur Genüge aus der Thatsache, dass die drüsigen Verästelungen des Gartner'schen Ganges in der Cervix anseheinend relativ häufig gefunden werden, paroophorale Urnierenabschnitte im Corpus uteri oder Tubenwinkel dagegen bisher überhaupt noch nicht sicher nachgewiesen sind.

Dann aber lehrt auch dic Morphologie der Schläuche an sich ganz klar, dass sie zu irgend welchen paroophoralen Urnierenbildungen nicht die allergeringste Beziehung haben können.

Dass keine Urnierenglomeruli oder Reste derselben an ihnen nachweisbar sind, fiele füglich nicht allzu schwer ins Gewicht. Denn am Genitale weiblicher Individuen sind bekanntlich im extraembryonalen Leben Malpighi'sche Körperchen oder sichere Degenerationsformen derselben bisher nicht gefunden. Keinesfalls aber passen die reichlichen, durchaus lebenskräftigen Schläuche und Epithelstränge zu dem übereinstimmend beobachteten und sichergestellten Factum, dass der Schwund des Wolff'schen Körpers distalwärts am vollkommensten ist, so vollkommen, dass schon das Paroophoron Waldeyer's in der Ala keine regelmässig nachweisbare Bildung mehr darstellt (vergl. z. B. v. Recklinghausen, 1, S. 119, 151). Ist es erhalten, so sieht man (Waldeyer, 16, S. 142) nicht mehr als "mehrere schmale mit epithelialen Zellen und körnigem Zelldetritus gefïllte" dürftige Canälchen!

Kurz: die nicht selten zu findenden cervicalen drüsigen Anhänge der Gartner'schen Gänge haben mit den Blinddärmchen des Wolff'schen Körpers nicht das Geringste zu thun.

Wie das Epoophoron der Epididymis, das Paroophoron den Vasa aberrantia des Nebenbodens und dem Giraldès'schen Organ, der Gartner'sche Gang selbst dem Vas deferens des Mannes entspricht, so bilden seine cervicalen Anhangsgebilde die Analoga der Samenbläschen und die von diesen distal gelegenen mehr oder minder erhaltenen vaginalen Abschnitte der Gänge die Analoga der männlichen Ductus ejaculatorii. Das hat z. B. Rieder in seiner These 4 (24, S. 129) kurz und präcis ausgesprochen.

Beim Mann, wo im Sinus pocularis die distalen Enden der Müller'schen Gänge in rudimentärer Form sich erhalten, lagern 
die nur contimeterlangen. Ductus als Ende und die Samonbläschen als Anhang der Ampullen der Samenleiter dicht beisammen.

Beim Weibe werden dagegen durch das starke Längenwachsthum des untersten Abschnitts der Müller'schen Gänge bei der Bildung der Scheide die dicht neben (und aussen) mit shnen zum Sinus urogenitalis tretenden Wolff'schen Gänge gestreckt, ja, gewöhnlich im distalen Ende völlig atrophirt, so dass z. B. Dohrn and Rieder (25 und 24, S. 111 und S. 124) in der Scheidenwand den Gartner'sehen Gang nie unterhalb der Nitte der Harnröhre zu Gesicht bekamen.

Es wird also hier der dem Ductus ejaculatorius entsprechende Theil des Wolff'schen Ganges zugleich mit dem correspondirenden Abschnitt des Müller'schen in die Länge gezogen, während die proximal zu ersterem gelegenen verzweigten Anhänge des Ganges - die Samenblasenanaloga _- in ihrer ursprünglichen Lagebeziehung beharren, mithin in die Seitentheile der Cervix uteri eingeschlossen werden.

Somit sind Adenomyome, Adenocystome oder Cystomyome, die von dem cervicalen Drüsenanhang des Gartner'schen Ganges ausgehen, von den v. Recklinghausenschen paroophoralen Adenocystomyomen genetisch genau so zu trennen, wie Adenocystome oder überhaupt Neoplasmen der Samenblasen von solehen des Nebenbodens, der Vasa aberrantia und des Giraldès'schen Organs.

Diese Trennung ist nicht von nur theoretischem Werth, insofern einige Fälle adenoeystomatöser und adenomyomatöser Geschwülste des cervicalen drüsigen Anhangs des Gartner'schen Ganges bereits bekannt sind. So beschreibt R. Meyer (13a, S. 332 u. 333) zwei angeborene Adenome dieser Art in der Portio, v. Recklinghausen ( 1, S. 63 and $S .74)$ in seinen Beobachtungen 17 und 21 zugleich mit Tubenwinkeladenomyomen einmal ein kleines cervicales Cystadenom des linken und im anderen Fall je ein kleines Adenocystomyom des rechten und linken Gartnerschen Ganges. Bemerkenswerther Weise führen auch diese Adenocystomyome unter Umständen (Fall 21, S. 740.) cytogenes Gewebe.

Würden, was gelegentlich auch beim Mensehen vorkommt (s. o.), die drüsigen Anhänge der Gartner'schen Gänge auch noch ein Stück über die Cervix hinaus nach abwärts sich erstrecken, so würden diese oder Geschwulstproducte derselben, dem Verlauf des Gartnepschen Ganges entsprechend, jederseits im seitlichen Scheiden- 
gowölbe zu suchen sein. Denn der Gartner'sehe Gang biegt ju der Portio rückläufig um(Klein), um im Bogen scitlich über das Scheidengewölbe an die seitliche Scheidenwand zu gelangen; er stellt sich, wie Rieder (1. c. S. 125) sich ausdrückt, beim Uebertritt auf das Scheidengewölbe "im Uterus von links nach reehts".

Demgegenüber hebe ich hervor, dass in allen drei Fällen fornicaler Adenomyome in ganz typischer Weise der Sitz im hinteren Scheidengewölbe wiederkehrt, and in dieser typischen Localisation sehe ich den durchaus eindeutigen Hinweis für die Herleitıng ihrer epithelialen Bestandtheile vom Wolff'schen Körper.

Ich brauche hier nur auf die Eingangs (S. 464-468) gegebene Darstellung des embryonalen Incorporationsvorganges von Urmierencanälchen in die Substanz des Müller'schen Ganges hinzuweisen. Reicht der Wolf f'sche Körper abnorm weit gegen das hintere Körperende (den Sinus urogenitalis) hinab, oder ist die Rückbildung der terminalsten, vielleicht besonders kräftigen Abschnitte noch nicht völlig vollzogen, wenn der Müller'sche Gang auf seinem Herabtritt zum Sinus urogenitalis nach ventraler Kreuzung des Wolff'schen Ganges diese Höhe passirt, so erfolgt Einschluss von Wolff'sehen (paroophoralen) Canälchen in das Dorsum des untersten Mülle r'schen Gangabschnitts, d. h. in die Dorsalwand der späteren Scheide. Umgekehrt: der Situs von Adenomyomen im Scheidendorsum spricht bei so ausgesprochener Constanz beweisend für die Ableitung der adenocystomatösen Bestandtheile von den untersten Quereanälchen der $\mathrm{Ur}_{\mathrm{r}}$ niere, sei es, dass diese Tumoren stets im hinteren Fornix oder auch gelegentlich in tieferen Abschnitten des Scheidendorsums vorkommen. Nach den bisherigen, im auffallenden Typus sich gIeichenden Beobachtungen von Adenomyomen der Scheide erscheint der Schluss gerechtfertigt, dass für die vaginalen paroophoralen Adenomyome der hintere Fornix zum mindesten den Lieblingssitz darstellt.

Ein zweiter nicht minder stringenter Beweis für die paroophorale Abstammung liegt aber ausserdem noch speciell bei unserem Fall im histologischen Aufbau der Drüsenbildungen der Adenomyome selbst.

Wie in den beiden oben citirten Gebärmutteradenomyomen (in v. Recklinghausen's Fall I und unserer eigenen Beobachtung) die Drüsenschläuche in Structur und gegenseitiger Verbindung mit den drüsigen Canälchen des Wolff'schen Organs weitgehend über- 
einstimmten, so stossen wir auch hier auf Formen, die zu demselben Vergleich nöthigen.

Ich hebe als wesentlichstes Moment die zierliche dichotomische Theilung hervor, die sowohl an den langen engen gleichcalibrirten Röhrchen der "Adern" wie an weiteren Räumen der "Inseln" in ausgedehntem Umfange zu beubachten ist (Tafel XXII, Fig. 1, Tafel XXIII, Fig. 3). Eine derartig reichliche fortgesetzte dichotomische Verästelung kommt bei gutartiger Wucherung von Producten der Müller'schen Gänge niemals vor. Dagegen ist sie das getreue Widerspiel der dichotomischen Verzweigung der Wolffschen Drüsencanäle und ihrer bleibenden Abkömmlinge, der Schläuche des Parovariums und des Giraldès'schen Organs (v. Recklinghausen, 1, S. 115, 117, 149). Auch v. R. sah gablige oder sternförmige Theilung und vielfache Zweigbildung an den geraden oder gewundenen Driisenschläuchen seines Uterusadenomyoms, wenn auch, wie aus seinen Beschreibungen und $A b-$ bildungen (Taf. V, Figg. 3, 4) hervorgeht, nicht in solcher Fülle wie in unserer Scheidengeschwulst. Hier treten (Fig. 3) in den weiten, gablig getheilten Hauptcanal von allen Seiten diè mit hohem Epithel versehenen engen langgestreckten Sammelröhren. Sie sind oft wieder getheilt und beginnen mit kugligen oder olivenförmigen Endkolben, die ein platteres Epithel führen. Zuweilen schliessen diese Endauftreibungen den Querschnitt kugliger 'Einstülpungen ein, die aus dichtem cytogenen Gewebe bestehen und von kubischen Epithelien überzogen sind ("Pseudoglomeruli“). Gelegentlich münden auch die Sammelröhren in einen weiteren Epithelraum parallel in "Kammform", bilden sammt diesem „ein Parovarium in verjüngtem Maassstabe". v. Recklinghausen hat (S. $113 \mathrm{ff}$.) die besondere Beziehung all dieser Eigenheiten zur Morphologie der Wolff'schen Canälchen eingehend auseinandergesetzt.

Die Adenomyome des hinteren Scheidengewölbes sind danach als "paroophorale" Adenomyome zu betrachten.

Jede andere Erklärung für die Herkunft ihres Epithels versagt, obschon eine ganze Reihe weiterer Hypothesen möglich ist: Ein versprengter Abschnitt des Müller'schen Ganges müsste - von anderen Einwänden abgesehen - plötzlich die Fähigkeit gewinnen, sich am Laquear vaginae dem Endometrium corporis gleich zu differenziren. - Ein primäres aterines oder iuxtauterines Adenomyom, das secundär an das Scheidengewölbe heranwanderte, ist 
wegen der continuirlichen Verbindung der Adenomyomsubstanz mit der Fornixmuseulatur uhne Weiteres auszuschliessen. - Scheidendrüsen sind in der Schleimhaut über den fornicalen Adenomyomen bei v. Herff und in unserem Fall nachgewiesen und wohl auch bei Pfannenstiel vorhanden. Sie haben indessen mit den Drüsen und Cystchen des Adenomyoms, wie v. Herff und Ito (9, S. 191, S. 1.92 und 9 a, S. 23) besonders hervorheben und wie dies auch für unseren Fall zutrifft, Nichts zo thun. Sie beschränken sich durchaus anf das Schleimhautstroma ${ }^{1}$ ), die Adenomyomdrüsen und -Cysten dagegen liegen „in der Tiefe", ja, werden (s. unseren Fall) mit zunehmender Tiefe reichlieher.

Am nächsten liegt es, die Entwicklung von Scheidendrüsen und den embryonalen Einschluss von paroophoralen Urnierenabschnitten im Scheidengewölbe als eine combinirte Missbildung aufzufassen. Denn einmal sind Scheidendrüsen als solche keine physiologischen Producte ${ }^{2}$, und zweitens hat v. Preuschen dieselben, allerdings meist in Form von Retentionscysten, gleichfalls vorwiegend an der hinteren Scheidenwand in der Nähe der Umschlagstelle des hinteren Scheidengewölbes beobachtet.

Gelegentlich können auch diese Scheidendrüsen an der Bildung von vaginalen Cystomyomen sich betheiligen, sei es mit (Fall v. Herff's), sei es ohne gleichzeitiges paroophorales Adenomyom. Im letzteren Fall würden solche Cystomyome nicht gerade stets im hinteren Fornix, aber doch gewöhnlich im oberen Theil der Seheide gefunden werden. Denn wesentlich in diesen sind, wie eben hervorgehoben, echte Drüsen beobachtet.

Ob sich Scheidendrüsen, adenomatöse Wucherungsproducte oder Cysten derselben mit jedem paroophoralen Scheidenadenomyom oder doch, wie es scheint, wenigstens mit jedem fornicalen paroophoralen Adenomyom combiniren, bleibt abzuwarten.

Endlich denkt Ito $(9 \mathrm{a}, \mathrm{S} .23)$ auch an Theile des Wolff-

1) Sie entsprechen in ihrem Aufbau bei v. Herff und in unserem Fall den Scheidendrüsen, wie sie zuerst v. Preuschen (27) geschildert hat. Bezüglich unserer Befunde (Infiltration, Blutung und Pigmentirung um die Drüsen) möchte ich busonders darauf hinweisen, dass auch v. Pr. in der Umgebung der Drüsen eine bedeutende Anhäufung weisser Blut- und Lymphkörperchen hin und wieder beobachtete.

2) Vergl, z. B. unter den neuesten Autoren Nagel, 20, S. 102; Pretti, 28. Schoidendrüsen stellen also keinen regelmässigen und jedenfalls sehr seltenen Befund dar. 
schen Ganges als Ausgang lür das Drüsenepithel dieser Tumoren, doch lässt sich irgend ein positives Beweismittel für eine solche Annahme wohl kaum beibringen.

Ueberhaupt ist ein Fall, in dem sich mit irgendwie nennenswerther Sicherheit Bestandtheile des Wolff'schen (Gartner'schen) Ganges selbst - in loco oder nach Verlagerung gewuchert - als Muttergewebe richtiger adenomatöser Geschwülste am Genitalschlauch bezeichnen liessen, meiner Meinung nach bisher nicht beobachtet. Wohl kennen wir Cysten des Wolff'schen Ganges, vielleicht auch einfache Cystomyome mit Erweiterung des Ganges in toto - Kossmann bestreitet (3, S. 379-381) übrigens auch diese - , aber keine "Adenome" desselben. Adenome des Wolffschen Ganges giebt es, wie ich gegenüber R. Meyer's (13a, S. 332 u., 333 , 335) Nomenclatur bemerke, weder in der Portio, noch im Myometrium überhaupt. Höchstens ist der Gang bei der Bildung der paroophoralen Adenomyome an den Wucherungsvorgängen insoweit betheiligt, als er vielleicht die "Hauptcanäle" liefert, also die Längsleisten der kleinen kammförmigen Parovarien.

Jedenfalls muss man Kossmann darin durchaus beipflichten, wenn er im Interesse des Verständnisses (3, S. 379) die Nothwendigkeit betont, bei der Erörterung der Adenomyomgenese am weiblichen Genitalcanal mehr, als das bisher geschehen ist (z. B. v. Recklinghausen, 1, S. 156), die Wucherungsproducte des Wolff'sehen Ganges und des Wolff'schen Körpers auseinanderzuhalten - und, wie ich hinzufügen möchte, die des cervicalen Drüsenanhangs der Wolff'schen Gänge.

Aus all'. diesen Darlegungen geht klar hervor, dass die Epithelien der Adenomyome der Leistenbeuge und des hinteren Scheidengewölbes schon allein wegen der eigenartigen Aufstellung dieser Tumoren auf den untersten paroophoralen Abschnitt des Wolff'schen Körpers zu beziehen sind. Distale Urnierenschläuche wurden hier eingeschlossen und widerstanden der physiologischen Atrophie, um später in neoplastischer Wucherung geringere oder grössere Bruchstrücke der embryonalen Drüse wieder hervorzubringen.

Damit wird gelegentlich ein zweiter wichtiger Indicator ihres Ursprungs geliefert: es entsteht eine besondere specifische Morphologie der adenomatösen Theile, welche die Einrichtungen des Wolff'schen Körpers wiederspiegelt.

Es ist leicht verständlich, dass lebenskräftig sich erhaltende 
Urnierencanälchen gelegentlich gleichzeitig sowohl zur Leistenbeuge wie auch zum hinteren Scheidendorsum gelangen, sich also die beiden Adenomyomarten combiniren können: man vergleiche den oben referirten Fall Pfannenstiel's.

Was folgt aus diesen Thatsachen für die Anschaung v. Recklinghausen's von der paroophoralen Abstammung seiner Uterusund Tubenadenomyome?

Da die paroophoralen Adenomyome der Leistengegend und des hinteren Laquear grösstentheils als Drüsenstroma ein richtiges cytogenes Bindegewebe führen, so ist damit das Vorkommen dieser Gewebsart für Neubildungsproducte des Wolff'schen Körpers bewiesen. Sofern auch die Adenomyome des cervicalen Drüsenanhangs des Gartner'schen Ganges (v. Recklinghausen, Fall 21 s. o.) cytogenes Bindegewebe führen, das der Cervixschleimhaut selbst bekanntlich fehlt, muss man fast auf eine allgemeine Neigung der Abzweigungen des Wolff'schen Ganges schliessen, bei adenomatöser Wucherung dieses Stroma zu erzengen.

Es kann also der Gehalt an cytogenem Bindegewebe als irgendwie beweiskräftiges Argument gegen die paroophorale Abstammung der uterinen Adenomyome fernerhin nicht geltend gemacht werden: dasselbe wird in zweifellos paroophoralen Adenomyomen gefunden, die nicht einmal im Uteruskörper liegen.

Da ferner in dem histologischen Aufbau der paroophoralen Adenomyome in Scheidenfornix und Leistengegend einerseits und der v. Recklinghausen'schen Adenomyome in Uterus- und Tubenwandung andererseits auch alle weiteren histologischen Besonderheiten, wie sie v. Recklinghausen umschrieben hat, sich decken - geschlossene und zerstrente Ordnung der Drüsenröhrchen, dic Inseln und Verbindungsschenkel (Adern), die isolirten Cystehen und halbmondförmigen Ampullen mit hohem Boden- und flachem Dachepithel, Flimmerepithelien, Neigung zu Blutung und Pigmentirung etc. -, die Entwicklungsgeschichte aber geradezu auf eine Incorporation von Paroophoroncanälchen in die Substanz des Müller'schen Ganges hinweist, so ist auch die Abkunft der adenocystomatösen Bestandtheile der v. Recklinghausen'schen Uterus- und Tubentumoren vom Wolff'schen Körper (Paroophoron) sehr wahrscheinlich. Ja, diese Wahrscheinlichkeit nähert sich der Sicherheit, weil die ganz auffällige, man kann sagen gesetzmässige Localisation dieser Geschwülste sich befriedigend nur durch die Annahme der Incorporation von Urnierencanälchen in den Müller- 
schen Gang erklären lässt, und ferner, weil es bei wie gewissen paroophoralen Adenomyomen des hinteren Fornix, so auch bei manchen des Uterus (Fall 1 v. Recklinghausen's, unser Fall) gelingt, an den drüsigen Structuren der Geschwülste specifische Uebereinstimmung mit dem Aufbau und der gegenseitigen Verbindung der Wolff'schen Canälchen zu entdecken.

Entsprechend ihrer genetischen Zusammengehörigkeit und sonstigen histologischen Uebereinstimmung mit den paroophoralen Adenomyomen der Uterus- und Tubenwandung lassen sich die Leistengegend- und hinteren Fornixadenomyome auch leicht in das allgemeine Schema einreihen, das v. Recklinghausen für seine Tumoren (1, III, S. $90 \mathrm{ff}$.) aufgestellt hat. Ja, sie wiederholen in ihrer gröberen Zusammensctzung im Wesentlichen genau die Eigenschaften der voluminösen subserösen und iuxtauterinen paroophoralen Adenomyome (Verf., 2), die ja gleichfalls ausserhalb der Wandungen des eigentlichen Genitalschlauches gelegen sind. Hängt doch "bis zu einem gewissen Grade mit dem Sitze der Adenomyome auch ihr Bat im Grossen zusammen".

Fornix- und Leistengegendadenomyome sind, wie die iuxtauterinen und subserösen, harte Adenomyome. Das Muskelgewebe überwiegt hier wie dort stark gegenüber dem drüsigen Bestandtheil. Durch keine präformirte Architectur geleitet, wächst die Muskelmasse in der irregulären Art des gewöhnlichen Fibromyoms. Eine Beziehung zum Verlauf der drüsigen Adern oder Inseln („moiréeschimmernde Scheiden") besteht nirgends. - Hier wic dort erfolgt das Wachsthum in einzelnen relativ gut umschriebenen knolligen Herden, die bei den fornicalen Adenomyomen aus dem Scheidenmuskelrohr heraus sich sowohl in's Parametrium, wie polypös in's Scheidenlumen vorwölben können. Zwar sind diese knolligen Wachsthumscentren bei den iuxtauterinen und subserösen Adenomyomen gewöhnlich in der Mehrzahl, bei den bisher bekannten, an sich nicht so voluminösen Adenomyomen der Leistengegend und des hinteren Fornix immer nur in der Einzahl vorhanden. In praktischer Beziehung aber folgt beide Male daraus genau das Gleiche: die Möglichkeit der isolirten Exstirpation der Geschwulst, unbeschadet eines etwas diffusen Zusammenhanges mit der Umgebung, der den Adenomyomen generell eigen ist.

Fin gewisser Unterschied zwischen den beiden Adenomyomgruppen liegt vielleicht allein darin, dass in den Fornix- und Lejstengegendadenomyomen nicht, wie in den iuxtauterinen und subserösen, 
dic Cystenbildung in den Vordergrund tritt und so auch die charakteristischen Höhlen und Höhlchen mit pigmentirtem Inhalt (v. Recklinghausen, 1, S. 99 u. S. 123) auf dem makroskopischen Querschnitt fehlen. Immerhin möchte ich auf die Möglichkeit hinweisen, dass gelegentlich auch grössere event. mit fibromyomatöser Wand versehene Cysten des hintern Fornix (oder überhaupt der Scheidenhinterwand) und der Leistengegend (des Ligamentum rotundum) aus paroophoralen Resten ihren Ursprung nehmen.

Im Uebrigen treten die Drüsen hier, wie in den iuxtauterinen und subserösen Adenomyomen sowohl in ggeschlossener" als "zerstreuter" Ordnung auf. Wahrscheinlich werden auch hier ursprünglich geschlossene Ordnungen durch die regellose Muskelproliferation zersplittert und überwuchert; in anderen Fällen (z. B. bei Bluhm) mag von vornherein eine nur geringe Proliferation der eingesprengten Schlätche stattgefunden haben.

Einige histologische Eigenheiten unseres Scheidenadenomyoms verdienen, verglichen mit den bisher bekannten Qualitäten der paroophoralen Adenomyome, besondere Erwähnung:

An Stelle hämorrhagisch zertrümmerter Inseln können knötchenförmige pigmentirte Herde auftreten, deren Bild durch die von der Umgebung her erfolgende Organisation sich besonders eigenartig gestaltet (S. o. S. 484). v. Recklinghausen sah (S. 99) "obsolescirte Endkolben" in Form kugliger diffus gelber Herde aus jugendlichem Binde- oder Schleimgewebe.

Die Beziehung der Adenomherdchen zu kleinen Blutgefässstämmehen, die v. Recklinghausen namentlich für die Tubenadenomyome feststellen konnte (1, S. 216 bezgl. S. 93, Abs. 2), ist in unserem fornicalen Adenomyom besonders augenfällig. Drüsigo Inseln werden von kleinen Arterien central durchbohrt oder sitzen ihnen seitlich wie Knospen an.

Specifische Urnierenformen sind unter den sechs genauer beschriebenen Fällen von Leisten- und Fornixadenomyomen nur in meinem Tumor und zwar, wie die Regel in derartigen Adenomyomfällen, nur an einzelnen Stellen vorhanden. In anderen Bezirken unserer Geschwulst und in zwei anderen dieser paroophoralen Adenomyome gleichen die mehr indifferenten Drüsen- und Cystenformen mit ihrem cytogenen Stroma durchans der Uterusschleimhaut, wie die Autoren selbst (Cullen, BIuhm) ausdrücklich betonen. BIuhm und Cullen erwähnen zwar auch "Ampullen" und "Pseudoglomeruli", Pfannenstiel ebenfalls einen "Pseudoglomerulus". 
Ich möchte indessen die Deutung dieser Befunde im Sinne der Urnierenstructur nicht zu hoch bemessen. Die irrige Auffassung Cullen's bezüglich seines "Pseudoglomerulus" ist bereits dureh Bluhm (8) gekennzeichnet. Er entspricht mit seiner "Kapsel" (6, Fig. 2 und Text dazu) allenfalls einem "Hauptcanal" v. Recklinghausen's.

Die Deutung irgendweleher Gebilde als "Pseudoglomeruli" in der Auffassung v. Recklinghausen's (1, S. 119) wird überhaupt meiner Meinung nach bei der grossen Häufigkeit aller möglichen "Papillenbildungen" am Boden zahlreicher grösserer und kleiner Drüsenräume am Besten nur da zugelassen, wo es sich ohne Zweifel um wirklich kuglige oder halbkuglige, an der Basis mit einer Einschnürung deutlich abgesetzte lymphadenoide Erhebungen aus der Cystenwand heraus handelt, und zwar namentlich dann, wenn sie in deutlichen Endkolbeneysten auftreten (L. Pick, 2, S. 168 und 0. S. 483 o. $)^{1}$ ).

Jedenfalls aber ergiebt sich aus dem directen Hinweis Cullen's und BJuhm's auf die morphologische Uebereinstimmung der adenomatösen Partien ihrer Tumoren mit der Uteruskörperschleimhaut auch für v. Recklinghausen's Tuben- und Uterusadenomyome, dass so wenig wie der Bestand an cytogenem Gewebe auch die Drüsenform, kurz, die Aehnlichkeit des Adenoms mit der Corpusmucosa, irgendwie positiv für die Herkunft der Epithelien der letzteren aus dem Müller'schen Gange spricht (z. B. Cullen, 6, S. 7; v. Lockstädt, 4, S. 27, Abs. 3), und andererseits nicht gegen die paroophorale Abstammung. Das seinerzeit von mir aufgestellte Gesetz, dass „zwischen neoplastischen Abkömmlingen der Mesonephros und der Müller'schen Gänge sich augenfällige Structuranalogien herausbilden können" ${ }^{2}$ ), ist zweifellos richtig. Eine Parovarialcyste kann von einer "Hydroparasalpinx" unter Umständen histologisch nicht unterschieden werden, und eine Adenominsel im Adenomyom, die "mikroskopisch der Uterusmucosa zum Verwechseln ähnlich sieht" (v. Loekstädt), kann deswegen doch aus einer Wucherung von Urnierenresten hervorgegangen sein.

1) Das kubische Epithel der Pseudoglomeruli (v. R., 1, S. 100) spricht übrigens, wie ich zu v. Recklinghausen's Ausführungen auf S. 120 bemerken möchte, keineswegs gegen den Vergleich mit Urnierenglomeruli. Denn auch hier ist das Epithel erst später platt (Weber, 22, S. 68), zuerst cylindrisch (S. 44); (vergl. auch Waldeyer bei v. Recklinghansen, 1, S.120u.).

2) 2, S. 120; vergl. auch Kossmann, 3, S. 373. 
So ist es, wie auch ich schon damals andeutete (1, S. 121), in der That nicht möglich, genau festzustellen, wie viele von den Tuben- oder Uterusadenomyomen oder auch der mehr isolirten drüsigen Einsprengungen, deren Epithelschläuche mit dem Endosalpingium oder Endometrium nicht in directer Continuität stehen oder andererseits specifischen Urnierenformen nicht gleichen, vom Müller'schen Gange und wie viele vom Wolff'schen Körper herstammen. Denn die blosse mehr centrale oder peripherische Lage zum Lumen des Genitalschlauchs ist weder nach der einen oder anderen Seite mit absoluter Sicherheit entscheidend. Können doch sogar z. B. zweifellos paroophorale Fornixadenomyome polypös in die Scheide hängєn! Immerhin deutet z. B. symmetrische Localisation von Adenomyomen an den Tubenwinkeln, subseröser Sitz am Dorsum oder in der peripherisehen Schicht des dorsalen Uterusscheitels eher auf paroophoralen als auf schleimhäutigen Ursprung:

Die Unmöglichkeit, die Genese gewisser Adenomyome zu erkennen, aber ändert Nichts an dem Hauptergebniss der obigen Ausführungen:

Die Drüsen und Cysten der Adenomyome der Leistengegend und des hinteren Scheidengewölbes entstammen sicher embryonal versprengten paroophoralen Abschnitten des Wolff'schen Körpers. Durch ihre besonderen Eigenschaften im Verein mit entwicklungsgeschichtlichen Gründen bilden sie wichtige Beweismittel für die Stichhaltigkeit der v. Recklinghausen'schen Anschaung vom paroophoralen Ursprung eines Theiles der Adenomyome der Uterus- und Tubenwandung.

Im Einzelnen schliesse ich folgendermaassen:

1. Die embryonale Incorporation von distalen (paroophoralen) Urnierencanälchen in den Müller'schen Gang, wie sie v. Recklinghausen. für die Genese seiner "Adenomyome der Uterusund Tubenwandung voraussetzt, ist auf Grund der Entwicklungsgeschichte leicht verständlich. Diese Annahme erklärt in ausgozeichneter Weise die Prädilectionsstellen der paroophoralen Adenomyome v. Recklinghausen's.

2. Die Unterscheidung der paroophoralen und schleimhäutigen Adenomyome des Uterus als peripherische und centrale Tumoren (v. Recklinghaùsen) ist höchstens im Allgemeinen aufrecht. zu erhalten. Paroophorale Adenomyome können bis an die Mu- 
cosa, schleimhäutige mit ihren Ausläufern bis an die Serosa herantreten.

3. Zu den von v. Recklinghausen aufgestellten Kategorien der paroophoralen und schleimhäntigen Adenomyome des Uterusund der Tubenwinkel sind neue makro- und mikroskopisch wohlcharakterisirte hinzuzufügen:

a) die voluminösen subserösen und iuxtanterinen Adenomyome;

b) die Kugelmyome des Uterus mit accidentellen Drüsenund Cysteneinsehlüssen;

c) die sehleimhäutigen Adenomyome der Tube.

4. Wie in der Uterus- und Tubenwandung giebt es auch in der Leistengegend und im hinteren Scheidengewölbe Adenomyomo. Die Adenomyome der Leistengegend sind gewöhnlich mit dem Ende. des runden Mutterbandes direct verbunden.

5. Die Epithelbestandtheile der Adenomyome der Leistengegend und des hinteren Scheidengewölbes sind von embryonal versprengten paroophoralen Abschnitten des Wolff'schen Körpers abzuleiten.

6. Für die Feststellung dieser Genese kommt in Betracht: in erster Linie die Localisation der Tumoren am Genitalschlauch, in zweiter die Structuranalogie der adenomatösen Bestandtheile einiger Geschwülste dieser Gruppe mit specifischen Urnierentheilchen. In letzterer Beziehung ist besonders die ausgesprochene dichtomische Verästelung hervorzuheben, die sich an Abzweigungen cinund desselben engeren oder weiteren Epithelrohres wiederholt.

7. Die paroophoralen Adenomyome der Leistengegend und des hinteren Fornix beweisen, dass um sichere Abkömmlinge von wuchernden Urnierencanälchen cytogenes Bindegewebe - und zwar ausserhalb des Uterus - producirt werden kann.

8. Da die Adenomyome der Uterus- und Tubenwandung $\mathrm{v}$. Recklinghausen's mit den eben genannten in allen histologischen Details übereinstimmen, so folgt daraus in Verbindung mit These 1 , dass die Theorie v. Recklinghausen's vom „paroophoralen" Ursprung der Uterus- und Tubenadenomyome für einen grossen Theil dieser Geschwülste eine sehr hohe Wahrscheinlichkeit besitzt.

Diese Wabrscheinlichkeit nähert sich der Sicherheit, weil die paroophoralen Adenomyome v. Recklinghausen's an Uterus und Tube sich gesetzmässig an denjenfigen Stellen finden, welche dic 
Scheidengewölbes, ihre Stellung zu den paroophor. Adenomyomen ete. 505

Theorie der paroophoralen Abstammung fordert, und weil gewisse Tumoren dieser Klasse specifische Urnierenformen enthalten.

9. In denjenigen paroophoralen Adenomyomen der Leistengegend und des hinteren Laquear vaginae, die keine specifischen Epithelformationen des Wolff'schen Körpers, wohl aber cytrgenes Bindegewebe (These 7) führen, gleichen die adenomatösen Bestandtheile durchaus dem Bilde der entzündlich veränderten Mucosa corporis uteri. Es können also Mesonephros und Müller'scher Gang in ihren Wucherungsproducten gleiche Structurbilder erzeugen.

10. Daraus folgt, dass:

a) die Aehnlichkeit der adenomatösen Theile mit entzündlich wuchernder Mucosa corporis uteri in vielen Uterus- und Tubenwinkeladenomyomen als Beweis für die Abstammung von dieser so wenig, wie gegen den paroophoralen Ursprung verwerthet werden kann;

b) falls nicht directe Continuität der Epithelschläuche des Adenomyoms mit der Uterus- oder Tubenschleimhaut besteht oder aber andererseits specifische Urnierenformen auftreten, eine sichere Grenze zwischen schleimhäutigen und paroophoralen Adenomyomen nicht immer gezogen werden kann: anatomisch indifferente Adenomyome. Denn. auch der centrale oder peripherische Sitz ist nach keiner Seite absolut charakteristisch (These 2). Sitz des Adenomyoms in den peripherischen Schichten des Uterusscheitels und Uterusdorsums, symmetrisches Auftreten an beiden Tubenwinkeln spricht zu Gunsten des paroophoralen Ursprungs.

11. Die Adenomyome der Leistengegend und des hinteren Scheidengewölbes sind harte Adenomyome im Sinne r. Recklinghausen's. Sie folgen in ihren anatomischen Eigenschaften dem Prototyp der iuxtauterinen und subserösen Adenomyome bis auf die ihnen fehlende Cystenbildung. Adenomyome des Scheidengewölbes entwickeln sich nicht nur als knotenförmige Verdickung der Fornixmusculatur in das Parametrium, sonderı auch polypös in die Scheide.

12. Cysten in der Leistengegend (im oder am Ligamentum rotundum) und der hinteren Scheidenwand, speciell am hinteron Fornix, die von einer einfachen Epithelschicht ausgekleidet sind und ev. glatte Muskelfasern in ihrer Wand enthalten, können aus paroophoralen Einschlüssen entspringen. 
13. Die bei Säugern, auch beim Menschen nicht seltenen "wurzelstockartigen" drüsigen Anhänge des Wolff'schen Ganges in der Cervix bilden die Analoga der Samenblasen, der distal von ihnen ev. vorhandene vaginale Abschnitt des Wolff'schen Ganges das Analogon des Ductus ejaculatorius.

14. Die Adenomyome und Cystadenome am cervicalen Drüsenanhang des Wolff'schen Ganges, die gleichfalls cytogenes Gewebe führen können, haben weder mit den Adenomyomen am hinteren Scheidengewölbe noch mit den Adenomyomen der Uterus- und Tubenwandung etwas zu thun. Alle diese auf der einen, die erstgenannten cervicalen Adenomyome auf der anderen Seite sind genetisch verschieden genau wie Cystadenome oder überhaupt Neubildungen der Samenblasen einerseits, und der Epi- oder Paradidymis andererseits.

15. Die bisher bekannten drüsen- und cystenführenden Myome am weiblichen Genitalapparat lassen sich unter Berücksichtigung ihres Situs, ihrer Abstammung und Structur in folgendem Schema übersichtlich zusammenfassen:

I. Uterus:

1. schleimhäutige Adenomyome (fötale Abschnürung oder postfötale Wucherung der Epithelien des Müller'schen Ganges):

A. Corpus; B. Cervix;

2. paroophorale Adenomyome (fötale Inclusion):

A. Corpus uteri:

a) harte $\langle\alpha)$ voluminöse; $\langle\alpha \alpha)$ intraparietale;

B. Tubenwinkel:

$$
\text { b) teleangiectatische; }
$$

a) harte;

b) teleangiectatische;

3. Cystadenome und Adenocystomyome des Drüsenauhangs des Gartner'schen Ganges: Cervix;

4. Kugelmyome mit accidentellen drüsigen paroophoralen oder schleimhäutigen Einschlüssen.

5. Cystomyome des Gartuer'schen Ganges.

II. Tube:

1. schleimhäutige Adenomyome;

2. paroophorale: mediale Eileiterhältte.

III. Scheide: 
Scheidengowölbes, ihre Stellung za den paroophor. Adenomyomen etc. 507

1. schleimhäutige Adenomyome;

2. paroophorale: hinterer Fornix; event. polypös.

IV. Ligamentum rotundum: paroophorale Adenomyome der Leistengegend; event. Abtrenuung rom Ligament.

V. Ligamentum latum:

1. paroophorale Adenomyome:

a) voluminöse iuxtauterine;

b) kleine.

2. Cystomyome des Gartner'schen Ganges.

\section{Literatur}

(ausser der in Fussnoten vermerkten).

1) v. Recklinghausen, Die Adenomyome und Cystadenome der Uterusund 'Tubenwandung, ihre Abkunft von Resten des Wolff'schen Körpers. Berlin 1896.

1a) W. A. Freund, Klinische Notizen zu den voluminösen Adenomyomen des Uterus. loc. cit. S. $220-226$.

2) L. Pick, Ein neuer Typus des voluminösen paroophoralen Adenomyoms; zugleich über eine bisher richt bekannte Geschwulstform der Gebärmutter (Adenomyoma psammopapillare) und über totale Verdoppelung des Eileiters. Dieses Archiv. Bd. 54. H. 1. S. 117 ff. 1897.

3) Kossmann, Die Abstammung der Drüsenschläuche in den Adenomyomen des Uterus und der Tuben. Eine kritische Studic. Dieses Archiv. Bd. 54. H. 2. S. $359 \mathrm{ff} .1897$.

4) v. Lockstaedt, Ueber Vorkommen und Bedeutung von Drüsenschlänchen in den Myomen des Uterus. Inaug. - Diss. Königsberg i. Pr. 1898. (Zugleich Monatsschr. f. Geburtsh. u. Gynäkol. Bd. 7. H. 2. S. $188 \mathrm{ff}$. Febr. 1898.).

5) ₹. Recklinghausen, Adenomyom des Ligam. rotund. Centralbl. $f$. allgem. Pathol. u. patholog. Anat. S. 862. 1896.

6) Cullen, Adenomyoma of the round Ligament. John Hopkins Hospital Bulletin. Mai und Juni 1896.

7) Pfannenstiel, Ueber die Adenomyome des Genitalstranges. Verhandl. d. deutschen Gesellsch. f. Gynäkol. S. $195 \mathrm{ff} .1897$.

8) Blubm, Zur Pathologie des Ligamentum rotundum. Dieses Archiv. Bd. 55. H. 3. S. 647 ff. 1898.

9) v. Herff, Ueber Cystomyome und Adenomyome der Scheide. Verhandl. d. deutschen Gesellsch. f. Gynäkol. S. $189 \mathrm{ff}$. 1897.

9a) Ito, Ueber Fibrome, Cystofibrome und Adenomyome der Scheide. Inaug.Diss. Halle 1897. 
10) Klein, Ueber die Beziehungen der Müller'schen zu den Wolfl'schen Gängen beim Weibe. Verhandl. d. deutschen Gesellsch. f. Gynäkol. S. 163 ff. u. S. 448.1897.

11) Gebhard, Anatomie und Histologie der Myome in Veit's Handbuch der Gynälrologie. Bd. 2. S. $405 \mathrm{ff} .1897$.

12) Orloff, Zur Genese der Uterusmyome. Prag. Zeitschr. f. Heilkunde. Bd. 16. S. $311 \mathrm{ff.} 1895$.

13) Ricker, Beitrag zur Aetiologie der Uterusgeschwülste. Virch. Arch. Bd. 142. S. $193 \mathrm{ff} .1895$.

13a) R. Meyer, Ueber die Genese der Cystadenomyome des Uterus. Zeitschr. f. Geburtsh. u. Gynäkol, Bd. 37. S. $327 \mathrm{ff} .1897$.

14) Rolly, Ueber einen Fall yon Adenomyoma uteri mit Uebergang in Carcinom und Metastasenbildung. Virch. Arch. Bd. 156. S. $555 \mathrm{ff} .1897$.

15) O. Hertwig, Lehrbuch der Entwicklungsgeschichte des Menschen und der Wirbelthiere. 1888.

16) Wieger, Ueber die Entstehung und Entwicklung der Bänder des weiblichen Genitalapparates beim Menschen. Ein Beitrag zur Lehre vom Descensus ovariorum. Arch. f. Anatom. u. Physiol. Anatom. Abtheil. S. $349 \mathrm{ff}, 1885$.

17) v. Mihálkovicz, Untersuchnngen über die Entwicklung des Harn- und Geschlechtsapparates der Amnioten. Internat. Monatsschr. f. Anatom. u. Histolog. Bd. 2. 1885.

18) Blumberg and Heymann, Ueber den Ursprung, den Verlauf und die Bedeutung der glatten Muskulatur in den Ligamenta lata beim Menschen und bei den Säugethieren. Arch. f. Anatom. u. Physiol. Anat. Abtheil. S. $264 \mathrm{ff} .1898$.

19) Klaatsch, Ueber den Descensus testiculorum. Morpholog. Jahrb. Bd. 16. S. $587 \mathrm{ff}, 1890$.

20) Nagel, Die weiblichen Geschlechtsorgane in K. v. Bardeleben's Handbuch der Anatomie des Menschen. Bd. 7. Th. 2. Abth. 1. 1896.

21) Derselbe, Ueber die Entwicklung des Urogenitalsystems des Menschen. Arch. f. mikrosk. Anatom. Bd. 34. S. 269 ff. 1889.

22) Weber, Zur Fntwiclingssgeschichte des uropoetischen Apparats bei Säugern mit besonderer Berücksichtigung der Uniere zur Zeit des Auftretens der bleibenden Niere. Inaug.-Diss. Freiburg 1897.

23) Peters. Die Urniere in ihrer Beziehung zur Gynäkologie. Volkmann's Sammlung klin. Vortr. No. 195. 1897.

24) Rieder, Ueber die Gartner'schen (Wolff'schen) Canäle beim menschlichen Weibe. Virch. Arch. Bd. 96. S. 100.

25) Dohru, Ueber die Gartner'schen Canäle beim Weibe. Dieses Archiv. Bd. 21. S. $328 \mathrm{ff.} 1883$.

26) Waldeyer, Eierstock und Ei. Ein Beitrag zur Anatomie und Entwicklungsgeschichte der Sexualorgane. Leipzig 1870.

27) v. Preuschen, Ueber Cystenbildung in derVagina. Virch. Arch. Bd. 70. S. $111 \mathrm{ff} .1877$.

28) Pretti, Beitrag zum Studium der histologischen Veränderungen der Scheide. Zeitschr. f. Geburtsh. u. Gynäkol. Bd. 38. H. 2. S. 250. 1898. 


\section{Erklärung der Abbildungen auf Tafel XXII und XXIII.}

Figg. 1, 2 und 3 entsprechen Stellen ans den centralen Partien des Fornixadenomyoms bei ca. 110 facher Vergrösserung ( $\mathrm{Z}$ eiss, Oc. 1, Obj. C.).

Figur 1. Ein anf langer Strecke zu verfolgender Canal (b), der mehrfach hintereinander dichotomisch getheilte gestreckte Röhrchen $\left(d_{1}, d_{2}\right)$ aufnimmt. (Bei verschiedener Einstellnng der Mikrometerschraube wird die Einmündung der letzteren in das Hauptrohr leicht sichtbar.) Einbettung der Schlänche in cytogenes Gewebe (cy) und stark bindegewebig durchseiztes glattes Muskelgewebe.

Figur 2. Eine langgestreokte "Insel" mit mehreren seitlich ansetzenden Aesten („Adern" oder „Inselschenkeln“) $a_{1}, a_{2}$, $a_{3}$. Schläuche der letzteren eng, gleichlaalibrirt, sanft gebogen, streckenweise parallel $\left(a_{3}\right)$. Röhren der Insel meist ausgeweitet, sinuös $\left(r_{1}, r_{2}\right)$; in $r_{2}$ eine quergetroffene papilläre Einragung.

Cytogenes Stroma (cy) in der "Insel" und ihren Ausläufern. Einbettendes Muskelgewebe (m) ohne Beziehung der Verlaufsrichtung seiner Bündel zur Schlauchriehtung.

Figur 3. Grosser und weiter längsgetroffener Canal (h) einer „Insel" mit zahlreichen, zum Theil dichotomisch ansetzenden Nebenzweigen $\left(s_{1}, s_{2}, s_{8}, s_{1}\right)$. Diese sind eng, stcllenweise parallel $\left(s_{2}, s_{3}\right)$ und beginnen zum Theil mit deutlich olivenförmigen (0) oder mehr unregelmässigen $\left(\mathrm{n}_{1}, \mathrm{u}_{2}\right)$ Anschwellungen. Einbettung des Systems in cytogene Bindesubstanz (cy) und regellos angeordnetes glattes Muskelgewebe (m).

Figur 4. Cervixquerschnitt von ausgetragenem todtgeborenen - Mädchen bei ca. 30 facher Vergr. Dr Cervixdrüsen. $m$ circuläre Cervixmuskulatur. G. G. Gartner'scher Gang. dr A Stroma und Drüsenanbang. Canälchen des Drüsenanhangs bis dicht an die Cervixdrüsen tretend. Deutliche Differenz der hohen hellen Cylinderzellen der letzteren mit den kurzen dunklen Epithelien der Schläuche des Drüsenanhangs. Weiteres im T'ext S. 492. 

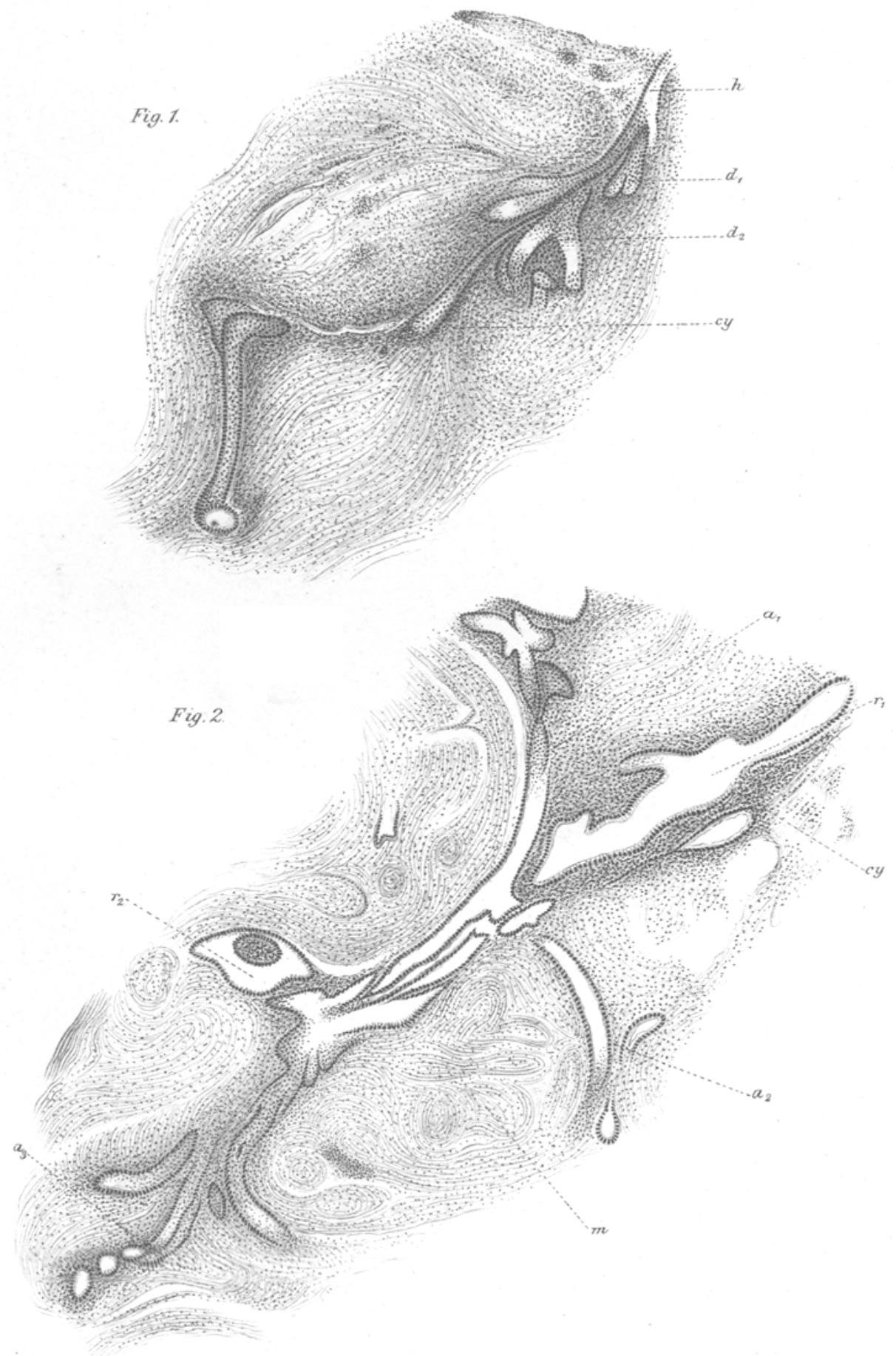


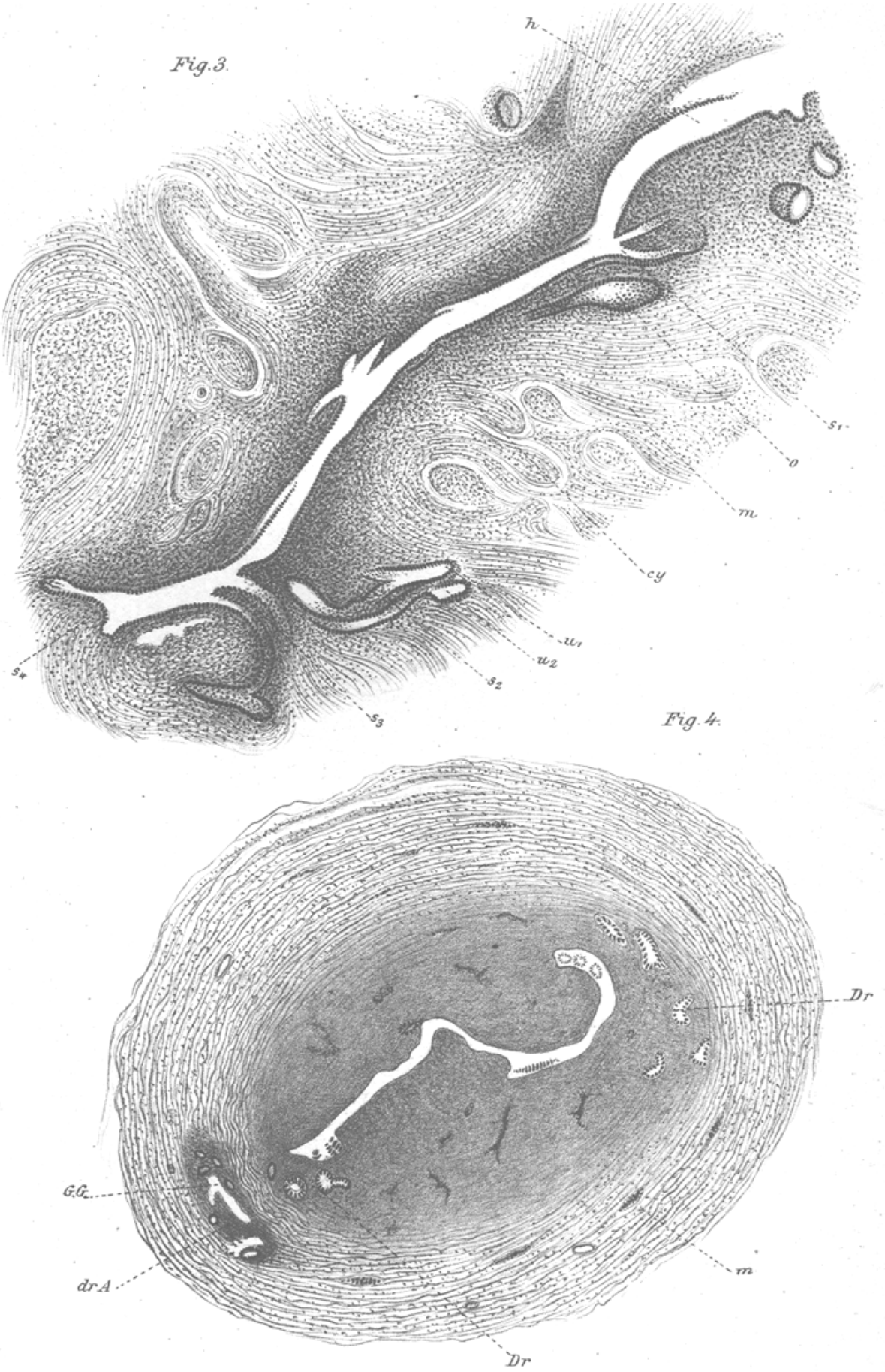

